\title{
Wavelet representation of light-front quantum field theory
}

\author{
W. N. Polyzou \\ Department of Physics and Astronomy, The University of Iowa, Iowa City, Iowa 52242, USA
}

(Received 28 January 2020; accepted 29 April 2020; published 11 May 2020)

\begin{abstract}
A formally exact discrete multiresolution representation of quantum field theory on a light front is presented. The formulation uses an orthonormal basis of compactly supported wavelets to expand the fields restricted to a light front. The representation has a number of useful properties. First, light-front preserving Poincaré transformations can be computed by transforming the arguments of the basis functions. The discrete field operators, which are defined by integrating the product of the field and a basis function over the light front, represent localized degrees of freedom on the light-front hyperplane. These discrete fields are irreducible and the vacuum is formally trivial. The light-front Hamiltonian and all of the Poincaré generators are linear combinations of normal ordered products of the discrete field operators with analytically computable constant coefficients. The representation is discrete and has natural resolution and volume truncations like lattice formulations. Because it is formally exact, it is possible to systematically compute corrections for eliminated degrees of freedom.
\end{abstract}

DOI: 10.1103/PhysRevD.101.096004

\section{INTRODUCTION}

A discrete multiresolution representation of quantum field theory on a light front is presented. Light-front formulations of quantum field theory have advantages for calculating electroweak current matrix elements in strongly interacting states in different frames. Lattice truncations have proved to be the most reliable method for nonperturbative calculations of strongly interacting states, but Lorentz transformation and scattering calculations are not naturally formulated in the lattice representation. The purpose of this work is to investigate a representation of quantum field theory that has some of the advantages of both approaches, although this initial work is limited to canonical field theory rather than gauge theories.

In 1939, Wigner [1] showed that the independence of quantum observables in different inertial reference frames related by Lorentz transformations and space-time translations requires the existence of a dynamical unitary representation of Poincare group on the Hilbert space of the quantum theory. Because there are many independent paths to the future, consistency of the initial value problem requires that a minimum of three of the infinitesimal generators of the Poincare group are interaction dependent. In 1949, Dirac [2] introduced three "forms of relativistic

Published by the American Physical Society under the terms of the Creative Commons Attribution 4.0 International license. Further distribution of this work must maintain attribution to the author(s) and the published article's title, journal citation, and DOI. Funded by SCOAP ${ }^{3}$. dynamics" that are characterized by having the largest interaction-independent subgroups.

Dirac's "front-form dynamics" is the only form of dynamics with the minimal number, 3, of dynamical Poincaré generators. The interaction-independent subgroup is the seven-parameter subgroup that leaves the hyperplane

$$
x^{+}=x^{0}+\hat{\mathbf{n}} \cdot \mathbf{x}=0
$$

invariant. The light-front representation of quantum dynamics has several advantages. One is that the kinematic (interaction-independent) subgroup has a three-parameter subgroup of Lorentz boosts. The subgroup property means that there are no Wigner rotations for light-front boosts. A consequence is that the magnetic quantum numbers of the light-front spin are invariant with respect to these boosts. A second advantage is that the boosts are independent of interactions. This means that boosts can be computed by applying the inverse transform to noninteracting basis states. These properties simplify theoretical treatments of electroweak probes of strongly interacting systems, where the initial and final hadronic states are in different Lorentz frames.

In light-front quantum field theory [3-14], there are additional advantages. These are consequences of the spectrum of the generator

$$
p^{+}=p^{0}+\hat{\mathbf{n}} \cdot \mathbf{p} \geq 0
$$

of translations in the

$$
x^{-}=x^{0}-\hat{\mathbf{n}} \cdot \mathbf{x}
$$


direction tangent to the light front. The first property is that free fields restricted to the light front are irreducible. This means that both the creation and annihilation operators for a free field can be constructed from the field restricted to the light front. It follows that any operator on the free-field Fock space can be expressed as a function of free fields restricted to the light front. The second advantage is that interactions that commute with the interaction-independent subgroup leave the Fock vacuum invariant. This means that it is possible to express all of the Poincare generators as operators on the free-field Fock space. There are ultraviolet and infrared $\left(p^{+}=0\right)$ singularities in the light-front Hamiltonian due to local operator products, which could impact these properties; however, in an effective theory with ultraviolet and infrared cutoffs, the interaction still leaves the Fock vacuum invariant and the light-front Hamiltonian can still be represented as a function of the free fields on the light front.

Having an explicit vacuum along with an expression for the light-front Hamiltonian,

$$
P^{-}=P^{0}-\mathbf{P} \cdot \mathbf{n},
$$

in terms of the algebra of fields on the light front means that it is possible to perform nonperturbative calculations by diagonalizing the light-front Hamiltonian in the light-front Fock space.

In a given experiment, there is a relevant volume and a finite amount of available energy. The available energy limits the resolution of the accessible degrees of freedom. The number of degrees of freedom with the limiting resolution that fits in the experimental volume is finite. It follows that it should be possible to accurately calculate experimental observables using only these degrees of freedom.

Wavelets can be used to represent fields on the light front as linear combinations of discrete field operators with different resolutions. This provides a natural representation to make both volume and resolution truncations consistent with a given reaction. In addition, the representation is discrete, which is a natural representation for computations. Finally, the basis functions are self-similar, so truncations with different resolutions have a similar form.

There are many different types of wavelets that have been discussed in the context of quantum field theory [1536]. The common feature is that the different functions have a common structure related by translations and scale transformations. This work uses Daubechies' wavelets [37-41]. These have the property that they are an orthonormal basis of functions with compact support. The price paid for the compact support is that they have a limited smoothness. It is also possible to use a wavelet basis of Schwartz functions that are infinitely differentiable, but these functions do not have compact support.
This work is an extension to the light front of the wavelet representations of quantum field theory used in Refs. [25,28,35]. The notation and development of the wavelet bases is identical to the development in these references. The difference is that the algebra generated by the discrete fields and conjugate generalized momenta in these papers is replaced by the irreducible algebra of fields on a light front. The light-front representation is formally exact and has all of the advantages of any other representation of light-front field theories.

There are several motivations for considering this approach. These include the following:

(1) Volume and resolution truncations can be performed naturally, the resulting truncated theory is similar to a lattice truncation $[42,43]$, in the sense that it is a theory involving a finite number of discrete degrees of freedom associated with a given volume and resolution.

(2) While the degrees of freedom are discrete, the field operators have a continuous space-time dependence. Kinematic Lorentz transformations can be computed by transforming the arguments of the basis functions. While truncations necessarily break kinematic Lorentz invariance, kinematic Lorentz transformations can still be approximated by transforming the arguments of the basis functions.

(3) Even though some truncations may lead to states with energy below the Fock vacuum energy, the error in using the free Fock vacuum as the lowest mass state of the truncated theory is due to corrections that arise from the discarded degrees of freedom.

(4) Since the representation is formally exact and $x^{+}$is a continuous variable, there is a formulation of HaagRuelle [44-46] scattering in this representation. Approximation methods need to be developed in the presence of truncations.

Some of the possible applications of the wavelet representation are discussed in [25] in the context of canonical field theory. There are a number of applications involving free fields that are straightforward and should be instructive. The advantage of free fields is that they can be solved and used as a testing ground in order to get an initial understanding of the convergence of truncated theories. One such application is understanding the restoration of Poincare invariance in truncated theories as the resolution is improved. An advantage of the wavelet representation is that this can be checked locally, i.e., in a small volume [25]. Understanding the restoration of Lorentz invariance is important for approximating current matrix elements. Another application involving free fields is to test the convergence of free-field commutator functions or Wightman functions based on truncated fields to the exact expressions. These can be approximated by iterating the Heisenberg field equations, which are simple in the 
free-field case. This could provide some insight into the nature of convergence in interacting theories. In [35], flow equation methods are used to block diagonalize the Hilbert space of a truncated free-field theory by resolution, constructing an effective Hamiltonian that involves only coarse-scale degrees of freedom, but includes the dynamics of the eliminated degrees of freedom. This calculation provided some insight into the complementary roles played by volume and resolution truncations.

While the elementary calculations discussed above can provide insight into the nature of approximations, the longterm goal is to use the wavelet representation to perform calculations of observables in 3+1-dimensional field theories. Calculations in $3+1$ dimensions are considerably more complicated for interacting theories. One computational method is to use the fields to construct a basis by applying discrete operators to the vacuum and then diagonalizing the light-front Hamiltonian in that basis. The light-front representation has the advantage that it is not necessary to first solve the vacuum problem. This method should be useful for modeling composite states that are spatially localized. This Hamiltonian approach is in the same spirit as the basis light-front quantization approach used in [47]. Variational methods could also be employed for low-lying composite states. Another method that takes advantage of the discrete nature of the wavelet representation is to use the light-front Heisenberg equations to generate an expansion of the field as a linear combination of products of fields restricted to the light front. Correlation functions can be computed by evaluating products of these fields in the light-front vacuum. In this case, while the algebra is discrete, the number of terms grows with each iteration. One of the advantages of the wavelet representation is that interactions involving different modes are self-similar and differ only by multiplicative scaling coefficients. A detailed study of the scaling properties could help to formulate efficient approximations to the solution of the light-front Heisenberg field equations by eliminating irrelevant degrees of freedom. Another potential use of the wavelet representation would be in quantum computing. In the wavelet representation, the field is replaced by discrete modes that only interact locally. This allows evolution over short time steps to be represented by quantum circuits involving products of local interactions.

This paper consists of 13 sections. The next section introduces the notation that will be used in this work, defines the light-front kinematic subgroup and the Poincaré generators that generate both the kinematic and dynamical Poincaré transformations. Section III discusses the irreducibility of free fields on the light front and properties of kinematically invariant interactions. Section IV discusses the structure of Poincare generators on the light front using Noether's theorem. The wavelet basis is constructed from the fixed point of a renormalization group equation in Sec. V. Wavelet representations of fields restricted to the light front are defined in Sec. VI. Section VII has a short discussion on kinematic Poincaré transformations of the fields in the light-front representation. In Sec. VIII, the irreducibility of the light-front free-field algebra and the triviality of the light-front vacuum are used to construct vectors in the light-front Fock space. Dynamical equations in the light-front wavelet representation are discussed in Sec. IX. Dynamical computations require expressions for the commutator of discrete fields on the light front, which are computed in Sec. X. In Sec. XI, the coefficients of the expansion of all ten Poincaré generators as polynomials of discrete fields on the light front are computed. Section XII discusses truncations and Sec. XIII gives a summary and outlook.

\section{NOTATION}

The light front is a three-dimensional hyperplane that is tangent to the light cone. It is defined by the constraint

$$
x^{+}:=x^{0}+\hat{\mathbf{n}} \cdot \mathbf{x}=0 .
$$

It is natural to introduce light-front coordinates of the fourvector $x^{\mu}$,

$$
x^{ \pm}:=x^{0} \pm \hat{\mathbf{n}} \cdot \mathbf{x}, \quad \mathbf{x}_{\perp}=\hat{\mathbf{n}} \times(\mathbf{x} \times \hat{\mathbf{n}}) .
$$

The components

$$
\tilde{\mathbf{x}}:=\left(x^{-}, \mathbf{x}_{\perp}\right)
$$

are coordinates of points on the light-front hyperplane. This will be referred to as a light-front three-vector. In what follows, the light front defined by $\hat{\mathbf{n}}=\hat{\mathbf{z}}$ will be used.

The contravariant light-front components are

$$
x_{ \pm}=-x^{\mp} \quad x_{i \perp}=x_{\perp}^{i},
$$

and the Lorentz-invariant scalar product of two light-front vectors is

$$
\begin{aligned}
x \cdot y & :=-\frac{1}{2} x^{+} y^{-}-\frac{1}{2} x^{-} y^{+}+\mathbf{x}_{\perp} \cdot \mathbf{y}_{\perp} \\
& =\frac{1}{2}\left(x^{+} y_{+}+x^{-} y_{-}\right)+x^{1} y_{1}+x^{2} y_{2} .
\end{aligned}
$$

For computational purposes, it is useful to represent four vectors by $2 \times 2$ Hermitian matrices. The coordinate matrix is constructed by contracting the four-vector $x^{\mu}$ with the Pauli matrices and the identity

$$
\begin{aligned}
X=x^{\mu} \sigma_{\mu} & =\left(\begin{array}{ll}
x^{+} & x_{\perp}^{*} \\
x_{\perp} & x^{-}
\end{array}\right) \quad x^{\mu}=\frac{1}{2} \operatorname{Tr}\left(\sigma_{\mu} X\right) \\
x_{\perp} & =x^{1}+i x^{2} .
\end{aligned}
$$

In this matrix representation, Poincaré transformations continuously connected to the identity are represented by

$$
X \rightarrow X^{\prime}=\Lambda X \Lambda^{\dagger}+A \quad \Lambda \in S L(2, \mathbb{C}) \quad A=A^{\dagger} .
$$


The subgroup of the Poincare group that leaves $x^{+}=0$ invariant consists of pairs of matrices $(\Lambda, A)$ in (11) of the form

$$
\Lambda=\left(\begin{array}{cc}
a & 0 \\
c & 1 / a
\end{array}\right) \quad A=\left(\begin{array}{cc}
0 & b_{\perp}^{*} \\
b_{\perp} & b^{-}
\end{array}\right),
$$

where $a, c$ and $b_{\perp}$ are complex and $b^{-}$is real. This is a seven-parameter group. The $S L(2, \mathbb{C})$ matrices with real $a$ represent light-front preserving boosts. They can be parametrized by the light-front components of the four velocity $v=p / m$,

$$
\begin{aligned}
\Lambda_{f}(p / m) & :=\left(\begin{array}{cc}
\sqrt{p^{+} / m} & 0 \\
\frac{p_{\perp} / m}{\sqrt{p^{+} / m}} & 1 / \sqrt{p^{+} / m}
\end{array}\right) \\
& =\left(\begin{array}{cc}
\sqrt{v^{+}} & 0 \\
v_{\perp} / \sqrt{v^{+}} & 1 / \sqrt{v^{+}}
\end{array}\right) .
\end{aligned}
$$

These lower triangular matrices form a subgroup. The inverse light-front boost is given by

$$
\begin{aligned}
\Lambda_{f}^{-1}(p / m) & :=\left(\begin{array}{cc}
1 / \sqrt{p^{+} / m} & 0 \\
-\frac{p_{\perp} / m}{\sqrt{p^{+} / m}} & \sqrt{p^{+} / m}
\end{array}\right) \\
& =\left(\begin{array}{cc}
1 / \sqrt{v^{+}} & 0 \\
-v_{\perp} / \sqrt{v^{+}} & \sqrt{v^{+}}
\end{array}\right),
\end{aligned}
$$

while the adjoint and the inverse adjoint of these matrices are

$$
\begin{aligned}
\Lambda_{f}^{\dagger}(p / m) & :=\left(\begin{array}{cc}
\sqrt{p^{+} / m} & \frac{p_{\perp}^{*} / m}{\sqrt{p^{+} / m}} \\
0 & 1 / \sqrt{p^{+} / m}
\end{array}\right) \\
& =\left(\begin{array}{cc}
\sqrt{v^{+}} & v_{\perp}^{*} / \sqrt{v^{+}} \\
0 & 1 / \sqrt{v^{+}}
\end{array}\right), \\
\left(\left(\Lambda_{f}\right)^{\dagger}\right)^{-1}(p / m) & :=\left(\begin{array}{cc}
1 / \sqrt{p^{+} / m} & -\frac{p_{\perp}^{*} / m}{\sqrt{p^{+} / m}} \\
0 & \sqrt{p^{+} / m}
\end{array}\right) \\
& =\left(\begin{array}{cc}
1 / \sqrt{v^{+}} & -v_{\perp}^{*} / \sqrt{v^{+}} \\
0 & \sqrt{v^{+}}
\end{array}\right) .
\end{aligned}
$$

General Poincaré transformations are generated by ten independent one-parameter subgroups. Seven of the oneparameter groups leave the light front invariant. The remaining 3 one-parameter groups map points on the light front to points off of the light front. These are called kinematic and dynamical transformations, respectively. The kinematic one-parameter groups in the $2 \times 2$ matrix representation and the corresponding unitary representations of these groups are related by

The corresponding dynamical transformations are

$$
\begin{gathered}
\Lambda(\lambda)=\left(\begin{array}{cc}
1 & \lambda \\
0 & 1
\end{array}\right) \quad U(\Lambda(\lambda))=e^{i F^{1} \lambda} \quad \Lambda(\lambda)=\left(\begin{array}{cc}
1 & -i \lambda \\
0 & 1
\end{array}\right) \quad U(\Lambda(\lambda))=e^{i F^{2} \lambda,} \\
A(\lambda)=\left(\begin{array}{ll}
\lambda & 0 \\
0 & 0
\end{array}\right) \quad U(\Lambda(\lambda))=e^{-\frac{i}{2} P^{-} \lambda} .
\end{gathered}
$$

Relations (17)-(20) define the infinitesimal generators

$$
\left\{P^{+}, P^{1}, P^{2}, E^{1}, E^{2}, K^{3}, J^{3}\right\}
$$


of the kinematic transformations, while (21) and (22) define the infinitesimal generators

$$
\left\{P^{-}, F^{1}, F^{2}\right\}
$$

of the dynamical transformations. With these definitions, the light-front Poincare generators are related to components of the angular-momentum tensor

$$
J^{\mu \nu}=\left(\begin{array}{cccc}
0 & -K^{1} & -K^{2} & -K^{3} \\
K^{1} & 0 & J^{3} & -J^{2} \\
K^{2} & -J^{3} & 0 & J^{1} \\
K^{3} & J^{2} & -J^{1} & 0
\end{array}\right)
$$

by

$$
E^{1}=K^{1}-J^{2} \quad E^{2}=K^{2}+J^{1} \quad F^{1}=K^{1}+J^{2} \quad F^{2}=K^{2}-J^{1} .
$$

The inverse relations are

$$
K^{1}=\frac{1}{2}\left(E^{1}+F^{1}\right) \quad K^{2}=\frac{1}{2}\left(E^{2}+F^{2}\right) \quad J^{1}=\frac{1}{2}\left(E^{2}-F^{2}\right) \quad J^{2}=\frac{1}{2}\left(F^{1}-E^{1}\right) .
$$

$F^{1}$ and $F^{2}$ could be replaced by $J^{1}$ and $J^{2}$ as dynamical generators.

The evolution of a state or operator with initial data on the light front is determined by the light-front Schrödinger equation

$$
i \frac{d\left|\psi\left(x^{+}\right)\right\rangle}{d x^{+}}=\frac{1}{2} P^{-}\left|\psi\left(x^{+}\right)\right\rangle
$$

or the light-front Heisenberg equations of motion

$$
\frac{d O\left(x^{+}\right)}{d x^{+}}=\frac{i}{2}\left[P^{-}, O\left(x^{+}\right)\right]
$$

When $P^{-}$is a self-adjoint operator, the dynamics is welldefined and given by the unitary one-parameter group (22).

The Poincaré Lie algebra has two polynomial invariants. The mass squared is

$$
M^{2}=P^{+} P^{-}-\mathbf{P}_{\perp}^{2},
$$

which gives the light-front dispersion relation

$$
P^{-}=\frac{M^{2}+\mathbf{P}^{2}}{P^{+}}
$$

The other invariant is the inner product of the PauliLubanski vector,

$$
W^{\mu}=\frac{1}{2} \epsilon^{\mu \nu \alpha \beta} P_{\nu} J_{\alpha \beta}
$$

with itself

$$
W^{2}=W^{\mu} W_{\mu}=M^{2} s^{2} .
$$

The Pauli-Lubanski vector has components

$$
W^{0}=\mathbf{P} \cdot \mathbf{J} \quad \mathbf{W}=H \mathbf{J}+\mathbf{P} \times \mathbf{K}
$$

or expressed in terms of the light-front Poincaré generators

$$
\begin{gathered}
W^{+}=P^{+} \mathbf{J} \cdot \hat{\mathbf{z}}+(\mathbf{P} \times \mathbf{E}) \cdot \hat{\mathbf{z}}, \\
\mathbf{W}_{\perp}=\frac{1}{2}\left(P^{+} \hat{\mathbf{z}} \times \mathbf{F}-P^{-} \hat{\mathbf{z}} \times \mathbf{E}\right)-(\hat{\mathbf{z}} \cdot \mathbf{K}) \hat{\mathbf{z}} \times \mathbf{P}, \\
W^{-}=P^{-} \mathbf{J} \cdot \hat{\mathbf{z}}-(\mathbf{P} \times \mathbf{F}) \cdot \hat{\mathbf{z}} .
\end{gathered}
$$

In order to compare the spins of particles in different frames, it is useful to transform both particles to their rest frame using an arbitrary but fixed set of Lorentz transformations parametrized by the four velocity of the particle. The light-front spin is the angular momentum measured in the particle's or system's rest frame when the particle or system are transformed to the rest frame with the inverse light-front preserving boosts (14),

$$
\begin{aligned}
\mathbf{s} \cdot \hat{\mathbf{z}} & =\mathbf{J} \cdot \hat{\mathbf{z}}-\frac{(\mathbf{E} \times \mathbf{P}) \cdot \hat{\mathbf{z}}}{P^{+}}=\frac{W^{+}}{P^{+}} \\
\mathbf{s}_{\perp} & =\left(\mathbf{W}_{\perp}-\mathbf{P}_{\perp} W^{+} / P^{+}\right) / M .
\end{aligned}
$$

The components of the light-front spin can also be expressed directly in terms of $J^{\mu \nu}$, 


$$
s^{i}=\frac{1}{2} \epsilon^{i j k}\left(\Lambda_{l f}^{-1}\right)^{j}{ }_{\mu}(P / M)\left(\Lambda_{l f}^{-1}\right)^{k}{ }_{\nu}(P / M) J^{\mu \nu},
$$

where in (40) the $P / M$ in the Lorentz boosts are operators.

\section{FIELDS}

Light-front free fields can be constructed from canonical free fields by changing variables $\mathbf{p} \rightarrow \tilde{\mathbf{p}}$, where $\tilde{\mathbf{p}}:=$ $\left(p^{+}, p^{1}, p^{2}\right)$ are the components of the light-front momentum conjugate to $\tilde{\mathbf{x}}$. The Fourier representation of a free scalar field of mass $m$ and its conjugate momentum operator are

$\phi(x)=\frac{1}{(2 \pi)^{3 / 2}} \int \frac{d \mathbf{p}}{\sqrt{2 \omega_{m}(\mathbf{p})}}\left(e^{i p \cdot x} a(\mathbf{p})+e^{-i p \cdot x} a^{\dagger}(\mathbf{p})\right)$,

$\pi(x)=-\frac{i}{(2 \pi)^{3 / 2}} \int d \mathbf{p} \sqrt{\frac{\omega_{m}(\mathbf{p})}{2}}\left(e^{i p \cdot x} a(\mathbf{p})-e^{-i p \cdot x} a^{\dagger}(\mathbf{p})\right)$,

where $\omega_{m}(\mathbf{p}):=\sqrt{m^{2}+\mathbf{p}^{2}}$ is the energy of a particle of mass $m, \mathbf{p}$ is its three-momentum, and $x \cdot p:=-\omega_{m}(\mathbf{p}) x^{0}+\mathbf{p} \cdot \mathbf{x}$.

Changing variables from the three momentum, $\mathbf{p}$, to the light-front components, $\tilde{\mathbf{p}}=\left(p^{+}, p^{1}, p^{2}\right)$, of the four momentum gives the light-front Fourier representation of $\phi(x)$,

$\phi(x)=\frac{1}{(2 \pi)^{3 / 2}} \int \frac{d p^{+} \theta\left(p^{+}\right)}{\sqrt{2 p^{+}}} d \mathbf{p}_{\perp}\left(e^{i p \cdot x} \tilde{a}(\tilde{\mathbf{p}})+e^{-i p \cdot x} \tilde{a}^{\dagger}(\tilde{\mathbf{p}})\right)$, where

$$
\begin{aligned}
& \left|\frac{\partial\left(p^{1}, p^{2}, p^{2}\right)}{\partial\left(p^{+}, p^{1}, p^{2}\right)}\right|=\frac{\omega_{m}(\mathbf{p})}{p^{+}} \\
& p \cdot x=-\frac{1}{2}\left(\frac{\mathbf{p}_{\perp}^{2}+m^{2}}{p^{+}} x^{+}+p^{+} x^{-}\right)+\mathbf{p}_{\perp} \cdot \mathbf{x}_{\perp}
\end{aligned}
$$

and

$$
\tilde{a}(\tilde{\mathbf{p}}):=\tilde{a}\left(p^{+}, \mathbf{p}_{\perp}\right)=a(\mathbf{p}) \sqrt{\frac{\omega_{m}(\mathbf{p})}{p^{+}}}
$$

It follows from

$$
\left[a(\mathbf{p}), a^{\dagger}\left(\mathbf{p}^{\prime}\right)\right]=\delta\left(\mathbf{p}-\mathbf{p}^{\prime}\right)
$$

and (44) and (45) that

$$
\left[a(\tilde{\mathbf{p}}), a^{\dagger}\left(\tilde{\mathbf{p}}^{\prime}\right)\right]=\delta\left(\tilde{\mathbf{p}}-\tilde{\mathbf{p}}^{\prime}\right)
$$

The spectral conditions

$$
\begin{gathered}
P^{ \pm}=H \pm P^{3}=\sqrt{M^{2}+\mathbf{P}^{2}} \pm P^{3} \geq 0 \\
P^{-}=\frac{M^{2}+\mathbf{P}^{2}}{P^{+}} \geq 0
\end{gathered}
$$

imply that it is possible to independently construct both $a(\tilde{\mathbf{p}})$ and $a^{\dagger}(\tilde{\mathbf{p}})$ from the field $\phi\left(x^{+}=0, \tilde{\mathbf{x}}\right)$ restricted to the light front. This can be done by computing the partial Fourier transform of the field on the light front,

$$
\phi\left(x^{+}=0, p^{+}, \mathbf{p}_{\perp}\right)=\frac{1}{(2 \pi)^{3 / 2}} \int e^{i p^{+} x^{-} / 2-i \mathbf{p}_{\perp} \cdot \mathbf{x}_{\perp}} \phi\left(x^{+}=0, x^{-}, \mathbf{x}_{\perp}\right) \frac{d \mathbf{x}_{\perp} d x^{-}}{2}
$$

The creation and annihilation operators can be read off of this expression,

$$
\begin{gathered}
\tilde{a}(\tilde{\mathbf{p}})=\sqrt{\frac{p^{+}}{2}} \theta\left(p^{+}\right) \phi\left(x^{+}=0, p^{+}, \mathbf{p}_{\perp}\right), \\
\tilde{a}^{\dagger}(\tilde{\mathbf{p}})=\sqrt{\frac{p^{+}}{2}} \theta\left(p^{+}\right) \phi\left(x^{+}=0,-p^{+}, \mathbf{p}_{\perp}\right) .
\end{gathered}
$$

Both operators are constructed directly from the field restricted to the light front without constructing a generalized momentum operator. This means that $\phi(x)$ restricted to the light front defines an irreducible set of operators. It follows that any operator $O$ on the Fock space that commutes with $\phi\left(x^{+}=0, \tilde{\mathbf{x}}\right)$ at all points on the light front must be a constant multiple of the identity,

$$
\left[\phi\left(x^{+}=0, \tilde{\mathbf{x}}\right), O\right]=0 \rightarrow O=c I
$$

An important observation is that the only place where the mass of the field appears is in the expression for the coefficient of $x^{+}$. When the field is restricted to the light front, $x^{+} \rightarrow 0$, all information about the mass (and dynamics) disappears.

This is in contrast to the canonical case because the canonical transformation that relates free canonical fields and their generalized momenta with different masses cannot be realized by a unitary transformation [48]. 
When these fields are restricted to the light front, they become unitarily equivalent [10]. This is because dynamical information that distinguishes the different representations is lost as a result of the restriction.
Since the fields restricted to the light front are irreducible, the canonical commutation relations are replaced by the commutator of the fields at different points on the light front,

$$
\begin{aligned}
{\left[\phi\left(x^{+}=0, \tilde{\mathbf{x}}\right), \phi\left(y^{+}=0, \tilde{\mathbf{y}}\right)\right]=} & \frac{i}{2 \pi} \int \frac{d p^{+} \theta\left(p^{+}\right)}{p^{+}} \frac{e^{-\frac{i}{2} p^{+}\left(x^{-}-y^{-}\right)}-e^{\frac{i}{2} p^{+}\left(x^{-}-y^{-}\right)}}{2 i} \delta\left(\mathbf{x}_{\perp}-\mathbf{y}_{\perp}\right)= \\
& -\frac{i}{2 \pi} \int \frac{d p^{+} \theta\left(p^{+}\right)}{p^{+}} \sin \left(\frac{1}{2} p^{+}\left(x^{-}-y^{-}\right)\right) \delta\left(\mathbf{x}_{\perp}-\mathbf{y}_{\perp}\right)=-\frac{i}{4} \epsilon\left(x^{-}-y^{-}\right) \delta\left(\mathbf{x}_{\perp}-\mathbf{y}_{\perp}\right) .
\end{aligned}
$$

Note that while the $x^{-}$derivative gives

$$
\frac{\partial}{\partial x^{-}}\left[\phi\left(x^{+}=0, \tilde{\mathbf{x}}\right), \phi\left(y^{+}=0, \tilde{\mathbf{y}}\right)\right]=-\frac{i}{2} \delta\left(x^{-}-y^{-}\right) \delta\left(\mathbf{x}_{\perp}-\mathbf{y}_{\perp}\right),
$$

$\partial_{-} \phi(x)$ is not the canonical momentum.

Interactions that preserve the light-front kinematic symmetry must commute with the kinematic subgroup. In particular, they must be invariant with respect to translations in the $x^{-}$direction. This means that the interactions must commute with $P^{+}$, which is a kinematic operator. Since $P^{+}=\sum_{i} P_{i}^{+}$is kinematic, the vacuum of the field theory is invariant with respect to these translations, independent of interactions. This requires that

$$
\left[P^{+}, V\right]=0 \quad P^{+}|0\rangle=0,
$$

which implies

$$
P^{+} V|0\rangle=V P^{+}|0\rangle=0,
$$

where $|0\rangle$ is the free-field Fock vacuum. This means that $V|0\rangle$ is an eigenstate of $P^{+}$with eigenvalue 0 . Inserting a complete set of intermediate states between $V^{\dagger}$ and $V$ in $\left\langle 0\left|V^{\dagger} V\right| 0\right\rangle$, the absolutely continuous spectrum of $p_{i}^{+}$ cannot contribute to the sum over intermediate states because $p_{i}^{+}=0$ is a set of measure 0 . This means that

$$
V|0\rangle=|0\rangle\langle 0|V| 0\rangle
$$

or interactions that preserve the kinematic symmetry leave the free-field Fock vacuum unchanged.

The observation that the interaction leaves the vacuum invariant implies that it is an operator on the free-field Fock space. The irreducibility of the light-front Fock algebra means that the interaction can be expressed in terms of fields in this algebra. The Poincaré generators, defined by integrating the + components of the Noether currents that come from Poincaré invariance of the action over the light front, are also linear in this interaction. This means that it should be possible to solve for the relativistic dynamics of the field on the light-front Fock space.
A more careful analysis shows that the interaction, while formally leaving the light-front invariant, has singularities at $p^{+}=0$, so the formal expressions for the interactiondependent generators are not well-defined self-adjoint operators on the free-field Fock space. This is because the interaction contains products of operator-valued distributions which are not defined. Discussions of the nontriviality of the light-front vacuum and the associated "zero-mode" problem, which is the subject of many papers, can be found in $[7,49-55]$ and the references cited therein.

The expressions for the Poincare generators are defined on the free-field Fock space if infrared and ultraviolet cutoffs are introduced, but the cutoffs break the Poincare symmetry. The nontrivial problem is how to remove the cutoffs in a manner that recovers the Poincare symmetry.

While the solution of this last problem is equivalent to the unsolved problem of giving a nonperturbative definition of the theory, cutoff theories should lead to good approximations for observables on scales where the cutoffs are not expected to be important.

\section{FORMAL LIGHT-FRONT FIELD DYNAMICS}

The Lagrangian density for a scalar field theory is

$\mathcal{L}(\phi(x))=-\frac{1}{2} \eta^{\mu \nu} \partial_{\mu} \phi(x) \partial_{\nu} \phi(x)-\frac{1}{2} m^{2} \phi(x)^{2}-V(\phi(x))$,

where $\eta^{\mu \nu}$ is the metric tensor with signature $(-,+,+,+)$. The action is

$$
A[V, \phi]=\int_{V} d^{4} x \mathcal{L}(\phi(x)) .
$$

Variations of the field that leave the action stationary satisfy the field equation, 


$$
\frac{\partial^{2} \phi(x)}{\partial\left(x^{0}\right)^{2}}-\nabla^{2} \phi(x)+m^{2} \phi(x)+\frac{\partial V(\phi)}{\partial \phi(x)}=0 .
$$

Changing to light-front variables, the partial derivatives become

$$
\begin{aligned}
& \partial_{0}:=\frac{\partial}{\partial x^{0}}=\frac{\partial x^{+}}{\partial x^{0}} \frac{\partial}{\partial x^{+}}+\frac{\partial x^{-}}{\partial x^{0}} \frac{\partial}{\partial x^{-}}=\frac{\partial}{\partial x^{+}}+\frac{\partial}{\partial x^{-}}=\partial_{+}+\partial_{-}, \\
& \partial_{3}:=\frac{\partial}{\partial x^{3}}=\frac{\partial x^{+}}{\partial x^{3}} \frac{\partial}{\partial x^{+}}+\frac{\partial x^{-}}{\partial x^{3}} \frac{\partial}{\partial x^{-}}=\frac{\partial}{\partial x^{+}}-\frac{\partial}{\partial x^{-}}=\partial_{+}-\partial_{-} .
\end{aligned}
$$

Squaring and subtracting give

$$
\frac{\partial^{2}}{\partial\left(x^{0}\right)^{2}}-\frac{\partial^{2}}{\partial\left(x^{3}\right)^{2}}=4 \frac{\partial}{\partial x^{+}} \frac{\partial}{\partial x^{-}} .
$$

It follows that the Lagrangian density (60) and the field equation in light-front variables have the forms

$$
\begin{aligned}
\mathcal{L}(\phi(x))= & 2 \partial_{-} \phi(x) \partial_{+} \phi(x)-\frac{1}{2} \nabla_{\perp} \phi(x) \cdot \nabla_{\perp} \phi(x) \\
& -\frac{1}{2} m^{2} \phi(x)^{2}-V(\phi(x))
\end{aligned}
$$

and

$$
4 \partial_{+} \partial_{-} \phi(x)-\nabla_{\perp}^{2} \phi(x)+m^{2} \phi(x)+\frac{\partial V(\phi)}{\partial \phi(x)}=0 .
$$

Invariance of the action under infinitesimal changes in the fields and coordinates

$$
\phi(x) \rightarrow \phi^{\prime}\left(x^{\prime}\right)=\phi(x)+\delta \phi(x) \quad x^{\mu} \rightarrow x^{\prime \mu}+\delta x^{\mu}(x),
$$

along with the field equation, leads to the conserved Noether currents,

$$
\partial_{\mu} J^{\mu}(x)=0,
$$

where the Noether current is

$$
J^{\mu}(x)=\mathcal{L} \eta^{\mu \nu} \delta x_{\nu}+\frac{\partial \mathcal{L}(\phi)}{\partial\left(\partial_{\mu} \phi\right)}\left(\delta \phi(x)-\partial_{\nu} \delta x^{\nu}\right) .
$$

The Noether currents associated with translational and Lorentz invariance of the action are the energy-momentum, $T^{\mu \nu}$, and angular-momentum, $M^{\mu \alpha \beta}$, tensors,

$$
\partial_{\mu} T^{\mu \nu}=0 \quad \partial_{\mu} M^{\mu \alpha \beta}=0,
$$

where for the Lagrangian density (66)

$$
\begin{gathered}
T^{\mu \nu}=\eta^{\mu \nu} \mathcal{L}(\phi(x))+\partial^{\mu} \phi(x) \partial^{\nu} \phi(x), \\
M^{\mu \alpha \beta}=T^{\mu \alpha} x^{\beta}-T^{\mu \beta} x^{\alpha} .
\end{gathered}
$$

Integrating the + component of the conserved current over the light front, assuming that the fields vanish on the boundary of the light front, gives the light-front conserved (independent of $x^{+}$) charges,

$$
\frac{d}{d x^{+}} P^{\mu}=0 \quad \frac{d}{d x^{+}} J^{\alpha \beta}=0,
$$

where

$$
P^{\mu}:=\int \frac{d \mathbf{x}_{\perp} d x^{-}}{2} T^{+\mu}=\int \frac{d \mathbf{x}_{\perp} d x^{-}}{2}\left(T^{0 \mu}+T^{3 \mu}\right)
$$

and

$$
\begin{aligned}
J^{\alpha \beta} & :=\int \frac{d \mathbf{x}_{\perp} d x^{-}}{2} M^{+\alpha \beta} \\
& =\int \frac{d \mathbf{x}_{\perp} d x^{-}}{2}\left(\left(T^{0 \alpha}+T^{3 \alpha}\right) x^{\beta}-\left(T^{0 \beta}+T^{3 \beta}\right) x^{\alpha}\right) .
\end{aligned}
$$

These are the conserved four-momentum and angularmomentum tensors. They are independent of $x^{+}$and thus can be expressed in terms of fields and derivatives of fields restricted to the light front.

In order to construct the Poincare generators, the first step is to express the + component of the energy-momentum tensor and angular-momentum tensors in terms of fields on the light front,

$$
\begin{gathered}
T^{++}=4 \partial_{-} \phi(x) \partial_{-} \phi(x), \\
T^{+i}=-2 \partial_{-} \phi(x) \partial_{i} \phi(x), \\
T^{+-}=\nabla_{\perp} \phi(x) \cdot \nabla_{\perp} \phi(x)+m^{2} \phi^{2}(x)+2 V(\phi(x)), \\
M^{++-}=4 \partial_{-} \phi(x) \partial_{-} \phi(x) x^{-}-\left(\nabla_{\perp} \phi(x) \cdot \nabla_{\perp} \phi(x)\right. \\
+m^{2} \phi^{2}(x)+2 V(\phi(x)) x^{+}, \\
M^{++i}=4 \partial_{-} \phi(x) \partial_{-} \phi(x) x^{i}+2 \partial_{-} \phi(x) \partial_{i} \phi(x) x^{+}, \\
M^{+-i}=\left(\nabla_{\perp} \phi(x) \cdot \nabla_{\perp} \phi(x)+m^{2} \phi^{2}(x)\right. \\
+2 V(\phi(x)) x^{i}+2 \partial_{-} \phi(x) \partial_{i} \phi(x) x^{-}, \\
M^{+i j}=-2 \partial_{-} \phi(x) \partial_{i} \phi(x) x^{j}+2 \partial_{-} \phi(x) \partial_{j} \phi(x) x^{i} .
\end{gathered}
$$

The Poincaré generators are constructed by integrating these operators over the light front, 


$$
\begin{gathered}
P^{+}=4 \int \frac{d x^{-} d^{2} x_{\perp}}{2} \partial_{-} \phi(x) \partial_{-} \phi(x), \\
P^{i}=-2 \int \frac{d x^{-} d^{2} x_{\perp}}{2} \partial_{-} \phi(x) \partial_{i} \phi(x), \\
P^{-}=\int \frac{d x^{-} d^{2} x_{\perp}}{2}\left(\nabla_{\perp} \phi(x) \cdot \nabla_{\perp} \phi(x)+m^{2} \phi^{2}(x)+2 V(\phi(x)),\right. \\
J^{+-}=\int \frac{d x^{-} d^{2} x_{\perp}}{2}\left(4 \partial_{-} \phi(x) \partial_{-} \phi(x) x^{-}-\left(\nabla_{\perp} \phi(x) \cdot \nabla_{\perp} \phi(x)+m^{2} \phi^{2}(x)+2 V(\phi(x)) x^{+}\right),\right. \\
J^{-i}=\int \frac{d x^{-} d^{2} x_{\perp}}{2}\left(\left(\nabla_{\perp} \phi(x) \cdot \nabla_{\perp} \phi(x)+m^{2} \phi^{2}(x)+2 V(\phi(x)) x^{i}+2 \partial_{-} \phi(x) \partial_{i} \phi(x) x^{-}\right),\right. \\
J^{i j}=\int \frac{d x^{-} d^{2} x_{\perp} x_{\perp}}{2}\left(4 \partial_{-} \phi(x) \partial_{-} \phi(x) x^{i}+2 \partial_{-} \phi(x) \partial_{i} \phi(x) x^{+}\right),
\end{gathered}
$$

For free fields, these operators can be expressed in terms of the light-front creation and annihilation operators (51) and (52) using the identities

$$
\begin{aligned}
\int \frac{d x^{-} d^{2} x_{\perp}}{2}: \phi(x) \phi(x) & :=\int \frac{\theta\left(p^{+}\right) d p^{+} d^{2} p_{\perp}}{p^{+}} \tilde{a}^{\dagger}(\tilde{p}) \tilde{a}(\tilde{p}), \\
\int \frac{d x^{-} d^{2} x_{\perp}}{2}: \partial_{-} \phi(x) \partial_{-} \phi(x) & :=\frac{1}{4} \int \theta\left(p^{+}\right) d p^{+} d^{2} p_{\perp} \tilde{a}^{\dagger}(\tilde{p}) p^{+} \tilde{a}(\tilde{p}), \\
\int \frac{d x^{-} d^{2} x_{\perp}}{2}: \partial_{-} \phi(x) \partial_{i} \phi(x) & :=-\frac{1}{2} \int \theta\left(p^{+}\right) d p^{+} d^{2} p_{\perp} \tilde{a}^{\dagger}(\tilde{p}) p^{i} \tilde{a}(\tilde{p}), \\
\int \frac{d x^{-} d^{2} x_{\perp}}{2}: \partial_{i} \phi(x) \partial_{i} \phi(x) & :=\int \frac{\theta\left(p^{+}\right) d p^{+} d^{2} p_{\perp}}{p^{+}} \tilde{a}^{\dagger}(\tilde{p})\left(p^{i}\right)^{2} \tilde{a}(\tilde{p}) .
\end{aligned}
$$

Using (91)-(94) in (84)-(90) gives the following expressions for the Poincaré generators for a free field in terms of the lightfront creation and annihilation operators:

$$
\begin{gathered}
P^{+}=\int d p^{+} d^{2} p_{\perp} \theta\left(p^{+}\right) \tilde{a}^{\dagger}(\tilde{p}) p^{+} \tilde{a}(\tilde{p}), \\
P^{i}=\int d p^{+} d^{2} p_{\perp} \theta\left(p^{+}\right) \tilde{a}^{\dagger}(\tilde{p}) p^{i} \tilde{a}(\tilde{p}) \\
P^{-}=\int d p^{+} d^{2} p_{\perp} \theta\left(p^{+}\right) \tilde{a}^{\dagger}(\tilde{p}) \frac{\mathbf{p}_{\perp}^{2}+m^{2}}{p^{+}} \tilde{a}(\tilde{p}), \\
J^{+-}=\int d p^{+} d^{2} p_{\perp} \theta\left(p^{+}\right) \tilde{a}^{\dagger}(\tilde{p})\left(p^{+}\left(-2 i \frac{\partial}{\partial p^{+}}\right)-x^{+} \frac{\mathbf{p}_{\perp}^{2}+m^{2}}{p^{+}}\right) \tilde{a}(\tilde{p}), \\
J^{+i}=\int d p^{+} d^{2} p_{\perp} \theta\left(p^{+}\right) \tilde{a}^{\dagger}(\tilde{p})\left(\left(p^{+}\left(i \frac{\partial}{\partial p^{i}}\right)-p^{i} x^{+}\right) \tilde{a}(\tilde{p}),\right.
\end{gathered}
$$




$$
\begin{gathered}
J^{-i}=\int d p^{+} d^{2} p_{\perp} \theta\left(p^{+}\right) \tilde{a}^{\dagger}(\tilde{p})\left(\frac{\mathbf{p}_{\perp}^{2}+m^{2}}{p^{+}}\left(i \frac{\partial}{\partial p^{i}}\right)-2 p^{i}\left(-i \frac{\partial}{\partial p^{+}}\right)\right) \tilde{a}(\tilde{p}), \\
J^{i j}=\int d p^{+} d^{2} p_{\perp} \theta\left(p^{+}\right) \tilde{a}^{\dagger}(\tilde{p})\left(p^{j}\left(-i \frac{\partial}{\partial p^{i}}-p^{i}\left(-i \frac{\partial}{\partial p^{j}}\right)\right) \tilde{a}(\tilde{p}) .\right.
\end{gathered}
$$

Since these are independent of $x^{+}$, the expressions with an explicit $x^{+}$dependence can be evaluated at $x^{+}=0$. These expressions lead to the following identifications:

$$
\begin{gathered}
J^{+-}=-2 K^{3} \quad J^{+1}=K^{1}-J^{2}=E^{1} \quad J^{+2}=K^{2}+J^{1}=E^{2}, \\
J^{-1}=K^{1}+J^{2}=F^{1} \quad J^{-2}=K^{2}-J^{1}=F^{2} .
\end{gathered}
$$

\section{WAVELET BASIS}

In this section, the multiresolution basis that is used to represent the irreducible algebra of fields on the light front is introduced. Wavelets provide a natural means for exactly decomposing a field into independent discrete degrees of freedom labeled by volume and resolution. In this representation, there are natural truncations that eliminate degrees of freedom associated with volumes and resolutions that are expected to be unimportant in modeling a given reaction.

While there are many different types of wavelets, this application uses Daubechies $[37,38] L=3$ wavelets. These are used to generate an orthonormal basis of functions with the following desirable properties: (1) all of the basis functions have compact support, (ii) there are an infinite number of basis functions with compact support inside of any open set, (iii) the basis functions have one continuous derivative, and (iv) polynomials of degree 2 can be pointwise represented by locally finite linear combinations of these basis functions.

In what follows, these basis functions will be used to decompose fields restricted to a light front into an infinite linear combination of discrete operators with arbitrarily fine resolutions. The advantage of the light-front representation is that the resulting discrete algebra is irreducible and the vacuum remains trivial.

For Lagrangians that are polynomials in the fields, in the wavelet representation all of the Poincaré generators can be formally expressed as polynomials in the discrete fields on the light front with coefficients that can be computed analytically. While the polynomials are finite degree, there are an infinite number of discrete field operators.

The construction of the wavelet basis starts with the fixed-point solution of the renormalization group equation

$$
s(x)=\sum_{l=0}^{2 L-1} h_{l} D T^{l} s(x),
$$

where

$$
D f(x):=\sqrt{2} f(2 x) \text { and } T f(x):=f(x-1)
$$

are unitary scale transformations and translations. The fixed point, $s(x)$, is a linear combination of a weighted sum of translates of itself on a smaller scale by a factor of 2 . The weights $h_{l}$ are constant coefficients chosen, so $s(x)$ satisfies

$$
\begin{array}{cl}
\int T^{m} s(x) T^{n} s(x)=\delta_{m n} & \text { and } \\
x^{k}=\sum_{n} c_{n}^{k} T^{n} s(x) k<L \quad \text { pointwise. }
\end{array}
$$

There are different weights $h_{l}$ for different values of $L$. The $L=3$ weights are the algebraic numbers in Table I. Solving (104) is analogous to finding a fixed point of a block spin transformation, except the averaging over blocks is replaced by a weighted average.

The solution of the renormalization group equation (104) is a fractal valued function that has compact support for $x \in[0,2 L-1]$. For $L=3$, the solution has one continuous derivative with support on the interval $[0,5]$. Since the scale can be changed by a general unitary scale transformation, a scale is fixed by the convention

TABLE I. Scaling coefficients for Daubechies $\mathrm{L}=3$ wavelets.

\begin{tabular}{ll}
\hline \hline$h_{0}$ & $(1+\sqrt{10}+\sqrt{5+2 \sqrt{10}}) / 16 \sqrt{2}$ \\
$h_{1}$ & $(5+\sqrt{10}+3 \sqrt{5+2 \sqrt{10}}) / 16 \sqrt{2}$ \\
$h_{2}$ & $(10-2 \sqrt{10}+2 \sqrt{5+2 \sqrt{10}}) / 16 \sqrt{2}$ \\
$h_{3}$ & $(10-2 \sqrt{10}-2 \sqrt{5+2 \sqrt{10}}) / 16 \sqrt{2}$ \\
$h_{4}$ & $(5+\sqrt{10}-3 \sqrt{5+2 \sqrt{10}}) / 16 \sqrt{2}$ \\
$h_{5}$ & $(1+\sqrt{10}-\sqrt{5+2 \sqrt{10}}) / 16 \sqrt{2}$ \\
\hline \hline
\end{tabular}




$$
\int s(x) d x=1
$$

Because $s(x)$ is fractal valued, it cannot be represented in terms of elementary functions; however, it can be exactly calculated at all dyadic rationals using the renormalization group equation (104). It can also be approximated by iterating the renormalization group equation starting with a seed function satisfying (107). The evaluation of $s(x)$ is not necessary because most of the integrals that are needed in field theory applications can be evaluated exactly using the renormalization group equation. The integrals can be expressed in terms of solutions of finite linear systems of equations involving the numerical weights $h_{l}$ in Table I.

The next step in constructing the wavelet basis is to construct subspaces of $L^{2}(\mathbb{R})$ with different resolutions defined by

$$
\mathcal{S}^{k}:=\left\{\left.f(x)\left|f(x)=\sum_{n} c_{n} D^{k} T^{n} s(x) \quad \sum_{n}\right| c_{n}\right|^{2}<\infty\right\} .
$$

The resolution is determined by the width of the support of these functions, which for $L=3$, is $5 \times 2^{-k}$. The functions

$$
s_{n}^{k}(x):=D^{k} T^{n}(x) s(x),
$$

for fixed $k$, are orthonormal, have compact support on $\left[2^{-k} n, 2^{-k}(n+5)\right]$, satisfy

$$
\int s_{n}^{k}(x) d x=2^{-k / 2}
$$

and are locally finite partitions of unity

$$
\sum_{n} 2^{k / 2} s_{n}^{k}(x)=1
$$

The subspace $\mathcal{S}^{k}$ is called the resolution $2^{-k}$ subspace of $L^{2}(\mathbb{R})$.

The scale transformation $D$ has the following intertwining properties with translations and derivatives:

$$
T D=D T^{2} \quad \text { and } \quad \frac{d}{d x} D=2 D \frac{d}{d x} .
$$

Applying $D^{k} T^{n}$ to the renormalization group equation, using (112), gives

$s_{n}^{k}(x)=\sum_{l=0}^{2 L-1} h_{l} D^{k+1} T^{2 n+l} s(x)=\sum_{l=0}^{2 L-1} h_{l} s_{2 n+l}^{k+1}(x)$,

which expresses every basis element of $\mathcal{S}^{k}$ as a finite linear combination of basis elements of $\mathcal{S}^{k+1}$ or

$$
\mathcal{S}^{k} \subset \mathcal{S}^{k+1} \text {. }
$$

This means that the lower resolution subspaces are subspaces of the higher resolution subspaces. The orthogonal complement of $\mathcal{S}^{k}$ in $\mathcal{S}^{k+1}$ is called $\mathcal{W}^{k}$,

$$
\mathcal{S}^{k+1}=\mathcal{S}^{k} \oplus \mathcal{W}^{k}
$$

Since $\mathcal{W}^{k} \subset \mathcal{S}^{k+1}$, orthonormal basis functions $w_{n}^{k}(x)$ in $\mathcal{W}^{k}$ are also linear combinations of the $s_{n}^{k+1}(x)$. These functions are defined by

$$
w_{n}^{k}(x)=D^{k} T^{n} w(x),
$$

where $w(x)$ is the "mother wavelet" defined by

$$
w(x):=\sum_{l=0}^{2 L-1} g_{l} D T^{l} s(x),
$$

and the coefficients $g_{l}$ are related to the weight coefficients $h_{l}$ by

$$
g_{l}=(-)^{l} h_{2 L-1-l} \quad 0 \leq l \leq 2 L-1 .
$$

The orthonormal basis functions $w_{n}^{k}(x)$ for $\mathcal{W}^{k}$ are called wavelets. Since $w_{n}^{k}(x)$ are finite linear combinations of the $s_{n}^{k+1}(x)$, they have the same number of derivatives as $s(x)$. $w_{n}^{k}(x)$ also has the same support as $s_{n}^{k}(x)$. Finally, it follows from (106) that

$$
\int x^{m} w_{n}^{k}(x)=0 \quad 0 \leq m<L .
$$

Equation (119) is equivalent to the condition (106). Equation (115) means that the wavelet subspace $\mathcal{W}^{k}$ consists of functions that increase the resolution of $\mathcal{S}^{k}$ from $2^{-k}$ to $2^{-(k+1)}$.

The inclusions (114) imply a decomposition of $\mathcal{S}^{k+n}$ into an orthogonal direct sum of the form

$$
\mathcal{S}^{k+n}=\mathcal{W}^{k+n-1} \oplus \mathcal{W}^{k+n-2} \oplus \cdots \oplus \mathcal{W}^{k} \oplus \mathcal{S}^{k},
$$

which indicates that the resolution of $\mathcal{S}^{k}$ can be increased to $2^{-k-n}$ by including additional basis functions in the subspaces $\left\{\mathcal{W}^{k+n-1}, \ldots, \mathcal{W}^{k}\right\}$. This can be continued to arbitrarily fine resolutions to get all of $L^{2}(\mathbb{R})$,

$$
L^{2}(\mathbb{R})=\mathcal{S}^{k} \oplus_{n=0}^{\infty} \mathcal{W}^{k+n}=\oplus_{n=-\infty}^{\infty} \mathcal{W}^{n}
$$

Since all of the subspaces are orthogonal, an orthonormal basis for $L^{2}(\mathbb{R})$ consists of

$$
\left\{s_{n}^{k}(x)\right\}_{n=-\infty}^{\infty} \cup\left\{w_{n}^{m}(x)\right\}_{n=-\infty, m=k}^{\infty}
$$


for any fixed starting resolution $2^{-k}$ or

$$
\left\{w_{n}^{k}(x)\right\}_{k, n=-\infty}^{\infty} .
$$

The basis (123) includes functions of arbitrarily large support, while the basis (122) consists of functions with support in intervals of width $2^{-l}(2 L-1)$ for $l \geq k$.

The basis (122) is used with $L=3$ Daubechies wavelets [37,38]. Locally finite linear combinations of the $L=3$ scaling functions, $s_{n}^{k}(x)$, can be used to pointwise represent polynomials of degree 2 . The wavelets, $w_{n}^{l}(x)$, are orthogonal to these polynomials. The $L=3$ basis functions have one continuous derivative.

\section{WAVELET REPRESENTATION OF QUANTUM FIELDS}

In what follows, the basis (122) is used to expand quantum fields restricted to a light front. It is useful to think of the starting scale $2^{-k}$ in (122) as the resolution that is relevant to experimental measurements. The higher resolution degrees of freedom are used to represent shorter distance degrees of freedom that couple to experimentalscale degrees of freedom.

The basis (122) can be used to get a formally exact representation of the field operators of the form

$$
\phi\left(\tilde{\mathbf{x}}, x^{+}\right):=\sum \phi_{l m n}\left(x^{+}\right) \xi_{l}\left(x^{-}\right) \xi_{m}\left(x^{1}\right) \xi_{n}\left(x^{2}\right) \quad \text { where } \phi_{l m n}\left(x^{+}\right)=\int d^{2} x_{\perp} d x^{-} \xi_{l}\left(x^{-}\right) \xi_{m}\left(x^{1}\right) \xi_{n}\left(x^{2}\right) \phi\left(\tilde{\mathbf{x}}, x^{+}\right),
$$

where $\xi_{l}$ are the basis functions,

$$
\xi_{l}(x) \in\left\{s_{n}^{k}(x)\right\}_{n=-\infty}^{\infty} \cup\left\{w_{n}^{m}(x)\right\}_{n=-\infty, m=k}^{\infty} .
$$

In what follows, the shorthand notation is used,

$$
\xi_{\mathbf{n}}(\tilde{\mathbf{x}}):=\xi_{n_{-}}\left(x^{-}\right) \xi_{n_{1}}\left(x^{1}\right) \xi_{n_{2}}\left(x^{2}\right) \quad \sum_{\mathbf{n}}=\sum_{n_{-}} \sum_{n_{1}} \sum_{n_{2}} .
$$

With this notation, (124) has the form

$$
\phi\left(\tilde{\mathbf{x}}, x^{+}\right):=\sum_{\mathbf{n}} \phi_{\mathbf{n}}\left(x^{+}\right) \xi_{\mathbf{n}}(\tilde{\mathbf{x}}),
$$

which gives a discrete representation of the field as a linear combination of discrete operators with different resolutions on the light front.

Each discrete field operator, $\phi_{\mathbf{n}}(0)$, is associated with a degree of freedom that is localized in a given volume on the light-front hyperplane. In addition, there are an infinite number of these degrees of freedom that are localized in any open set on the light front.

While the fields are operator valued distributions, that does not preclude the existence of operators constructed by smearing with functions that have only one derivative. Note that the support condition implies that the Fourier transform of the basis functions are entire.

\section{KINEMATIC POINCARÉ TRANSFORMATIONS OF FIELDS IN THE WAVELET REPRESENTATION}

Since this representation is formally exact, kinematic Poincaré transformations on the algebra of fields restricted to the light front can be computed by acting on the basis functions. This follows from the kinematic covariance of the field

$U(\Lambda, a) \phi\left(\tilde{\mathbf{x}}, x^{+}=0\right) U^{\dagger}(\Lambda, a)=\phi\left((\tilde{\mathbf{\Lambda}} \tilde{\mathbf{x}}+\tilde{\mathbf{a}}), x^{+}=0\right)$

for $(\Lambda, a)$ in the light-front kinematic subgroup. Using the discrete representation of the field on both sides of this equation gives the identity

$$
\begin{aligned}
& U(\Lambda, a) \sum_{\mathbf{n}} \phi_{\mathbf{n}}\left(x^{+}=0\right) \xi_{\mathbf{n}}(\tilde{\mathbf{x}}) U^{\dagger}(\Lambda, a) \\
& =\sum_{\mathbf{n}} \phi_{\mathbf{n}}\left(x^{+}=0\right) \xi_{\mathbf{n}}(\tilde{\boldsymbol{\Lambda}} \tilde{\mathbf{x}}+\tilde{\mathbf{a}}) .
\end{aligned}
$$

This shows that kinematic transformations can be computed exactly by transforming the arguments of the expansion functions.

The transformation property of the discrete field operators restricted to a light front follows from the orthonormality of the basis functions (129),

$$
U(\Lambda, a) \phi_{\mathbf{n}}\left(x^{+}=0\right) U^{\dagger}(\Lambda, a)=\sum_{\mathbf{m}} \phi_{\mathbf{m}}\left(x^{+}=0\right) U_{\mathbf{m n}}(\tilde{\mathbf{\Lambda}}, \tilde{\mathbf{a}}),
$$

where the matrix

$$
U_{\mathbf{m n}}(\tilde{\mathbf{\Lambda}}, \tilde{\mathbf{a}}):=\int d^{2} x_{\perp} d x^{-} \xi_{\mathbf{m}}(\tilde{\boldsymbol{\Lambda}} \tilde{\mathbf{x}}+\tilde{\mathbf{a}}) \xi_{\mathbf{n}}(\tilde{\mathbf{x}})
$$

is a discrete representation of the light-front kinematic subgroup.

This identity implies that in the wavelet representation kinematic Lorentz transformations on the fields can be 
computed either by transforming the arguments of the basis functions or by transforming the discrete field operators.

\section{STATES IN THE WAVELET REPRESENTATION}

Because the algebra of free fields restricted to the light front is irreducible and kinematically invariant interactions leave the Fock vacuum unchanged, the Hilbert space for the dynamical model can be generated by applying functions of the discrete field operators, $\phi_{\mathbf{n}}\left(x^{+}=0\right)$, to the Fock vacuum.

Smeared light-front fields can be represented in the discrete representation as linear combinations of the discrete field operators,

$\phi\left(f, x^{+}=0\right):=\sum_{\mathbf{n}} \int d^{2} x_{\perp} d x^{-} f(\tilde{\mathbf{x}}) \xi_{\mathbf{n}}(\tilde{\mathbf{x}}) \phi_{\mathbf{n}}\left(x^{+}=0\right)$.

Equation (132) can be expressed as

$$
\phi\left(f, x^{+}=0\right)=\sum_{\mathbf{n}} f_{\mathbf{n}} \phi_{\mathbf{n}}\left(x^{+}=0\right),
$$

where

$$
f_{\mathbf{n}}:=\int d^{2} x_{\perp} d x^{-} f(\tilde{\mathbf{x}}) \xi_{\mathbf{n}}(\tilde{\mathbf{x}}) .
$$

States can be expressed as polynomials in the smeared fields applied to the light-front Fock vacuum

$$
\sum c_{m_{1} \cdots m_{n}} \phi\left(f_{m_{1}}, 0\right) \cdots \phi\left(f_{m_{n}}, 0\right)|0\rangle
$$

This representation can be reexpressed as a linear combination of products of discrete fields applied to the Fock vacuum

$$
\sum c_{\mathbf{m}_{1} \cdots \mathbf{m}_{n}} \phi_{\mathbf{m}_{1}}(0) \cdots \phi_{\mathbf{m}_{n}}(0)|0\rangle \text {. }
$$

The inner product of two vectors of this form is a linear combination of $n$-point functions. For the free-field algebra, the $n$-point functions are products of two-point functions. The two-point functions have the form

$$
\langle 0|\phi(f, 0) \phi(g, 0)| 0\rangle=\int \frac{\theta\left(p^{+}\right) d p^{+} d^{2} p_{\perp}}{2 p^{+}} \tilde{f}(-\tilde{\mathbf{p}}) \tilde{g}(\tilde{\mathbf{p}}) .
$$

This integral is logarithmically divergent if the Fourier transforms of the smearing functions do not vanish at $p^{+}=0$. Since $p^{+}=0$ corresponds to infinite threemomentum, this requirement is that the smearing functions need to vanish for infinite three-momentum.
From (133) and (137), it follows that the inner product above is a linear combination of two-point functions in the discrete fields, $\phi_{\mathbf{n}}\left(x^{+}=0\right)$.

The basis functions $\xi_{m}(x)$ have compact support which implies that their Fourier transforms are entire functions of the light-front momenta $\tilde{\mathbf{p}}$. This means that they cannot vanish in a neighborhood of $p^{+}=0$; however, they can have isolated zeroes at $p^{+}=0$. For the wavelet basis functions, $w_{m}^{l}(x)$, the vanishing (119) of the first three moments of the $L=3$ wavelets implies that

$$
\begin{aligned}
& \tilde{w}_{m}^{l}\left(p^{+}\right)_{p^{+}=0}=\frac{1}{2 \pi^{1 / 2}} \int w_{m}^{l}\left(x^{-}\right) d x^{-}=0 \\
& \frac{d}{d p^{+}} \tilde{w}_{m}^{l}\left(p^{+}\right)_{p^{+}=0}=-\frac{1}{2 \pi^{1 / 2}} \int x^{-} w_{m}^{l}\left(x^{-}\right) d x^{-}=0, \\
& \frac{d^{2}}{d^{2} p^{+}} \tilde{w}_{m}^{l}\left(p^{+}\right)_{p^{+}=0}=-\frac{1}{2 \pi^{1 / 2}} \int\left(x^{-}\right)^{2} w_{m}^{l}\left(x^{-}\right) d x^{-}=0 .
\end{aligned}
$$

Since the Fourier transforms are entire, this means that they have the form $\tilde{w}_{m}^{l}\left(p^{+}\right)=\left(p^{+}\right)^{3} f_{m}^{l}\left(p^{+}\right)$where $f_{m}^{l}\left(p^{+}\right)$is entire. For the scaling function basis functions, $s_{m}^{k}(x)$, the normalization condition (111) gives

$\tilde{s}_{m}^{k}\left(p^{+}\right)_{p^{+}=0}=\frac{1}{2 \pi^{1 / 2}} \int s_{m}^{k}\left(x^{-}\right) d x^{-}=\frac{1}{2 \pi^{1 / 2}} 2^{-k / 2} \neq 0$.

These results imply that

$$
\left\langle 0\left|\phi_{\mathbf{m}}\left(x^{+}=0\right) \phi_{\mathbf{n}}\left(x^{+}=0\right)\right| 0\right\rangle
$$

is singular if both basis functions have scaling functions in the $x^{-}$variable, but are finite if at least one of the basis functions has a wavelet in the $x^{-}$variable.

Since the smearing functions, $f(\tilde{\mathbf{p}})$, should all vanish at $p^{+}=0$, the discrete representation will involve linear combinations of wavelets and scaling functions whose Fourier transforms all vanish at $p^{+}=0$. In computing these quantities, the linear combinations of scaling functions should be summed before performing the integrals. This can alternatively be done by including a cutoff near $p^{+}=0$, doing the integrals, adding the contributions, and then letting the cutoff go to zero.

\section{DYNAMICS}

The dynamical problem involves diagonalizing $P^{-}$on the free-field Fock space or solving the light-front Schrödinger (28) or Heisenberg equations (29). The two dynamical equations can be put in integral form 
$\Psi\left(x^{+}\right)|0\rangle=\Psi\left(x^{+}=0\right)|0\rangle-\frac{i}{2} \int_{0}^{x^{+}}\left[P^{-}, \Psi\left(x^{+\prime}\right)\right]|0\rangle d x^{+\prime}$

or

$O\left(x^{+}\right)=O\left(x^{+}=0\right)+\frac{i}{2} \int_{0}^{x^{+}} d x^{+\prime}\left[P^{-}, O\left(x^{+\prime}\right)\right]$,

where $\Psi\left(x^{+}=0\right)$ and $O\left(x^{+}=0\right)$ are operators in the lightfront Fock algebra.

The formal iterative solution of these equations has the structure of a linear combination of products of discrete fields, $\phi_{\mathbf{n}}(0)$, in the light-front Fock algebra with $x^{+}$dependent coefficients. What is needed to perform this iteration are the initial operators $\Psi\left(x^{+}=0\right)$ and $O\left(x^{+}=0\right)$ expressed as polynomials in the $\phi_{\mathbf{n}}(0)$, the expression for $P^{-}$as a polynomial in the $\phi_{\mathbf{n}}(0)$, and an expression for the commutator, $\left[\phi_{\mathbf{m}}(0), \phi_{\mathbf{n}}(0)\right]$, of the discrete fields on the light front.

\section{THE COMMUTATOR}

It follows from (55) that the commutator of the discrete fields is

$$
\left[\phi_{\mathbf{m}}(0), \phi_{\mathbf{n}}(0)\right]=-\frac{i}{4} \delta_{m_{1} n_{1}} \delta_{m_{2} n_{2}} \int \xi_{m^{-}}\left(x^{-}\right) \epsilon\left(x^{-}-y^{-}\right) \xi_{n^{-}}\left(y^{-}\right) d x^{-} d y^{-}
$$

Unlike the inner product, the commutator is always finite since both $\xi_{m^{-}}\left(x^{-}\right)$and $\xi_{n^{-}}\left(y^{-}\right)$have compact support.

The commutator (144) can be computed exactly using the renormalization group equations. The computation involves three steps. The first step is to express $\xi_{m^{-}}\left(x^{-}\right)$ and $\xi_{n^{-}}\left(y^{-}\right)$as linear combinations of scaling functions on a sufficiently fine common scale. The second step is to change variables, so the commutator is expressed as a linear combination of commutators involving integer translates of the fixed-point solution $s\left(x^{-}\right)$of the renormalization group equation. The last step is to use the renormalization group equation to construct a finite linear system relating the commutators involving integer translates of the $s\left(x^{-}\right)$.
Applying $D^{k} T^{n}$ to the renormalization group equation and the expression for $w(x)$ gives

$$
D^{k} T^{n} s(x)=\sum_{L=0}^{l} h_{l} D^{k+1} T^{2 n+l} s(x)
$$

and

$$
D^{k} T^{n} w(x)=\sum_{L=0}^{l} g_{l} D^{k+1} T^{2 n+l} s(x)
$$

These equations express $s_{n}^{k}(x)$ and $w_{n}^{k}(x)$ as linear combinations of the $s_{n}^{k+1}(x)$,

$$
s_{n}^{k}(x)=\sum_{l=0}^{2 L-1} h_{l} s_{2 n+l}^{k+1}(x)=\sum_{m=2 n}^{2 n+2 L-1} h_{m-2 n} s_{m}^{k+1}(x)=\sum_{m=2 n}^{2 n+2 L-1} H_{n ; m} s_{m}^{k+1}(x) \quad \text { where } H_{n ; m}:=h_{m-2 n}
$$

and

$$
w_{n}^{k}(x)=\sum_{l=0}^{2 L-1} g_{l} s_{2 n+l}^{k+1}(x)=\sum_{m=2 n}^{2 n+2 L-1} g_{m-2 n} s_{m}^{k+1}(x)=\sum_{m=2 n}^{2 n+2 L-1} G_{n ; m} s_{m}^{k+1}(x) \quad \text { where } G_{n ; m}:=g_{m-2 n} .
$$

While the matrices $H_{n ; m}$ and $G_{n ; m}$ are formally infinite, for each fixed $n$, these are 0 unless $2 n \leq m \leq 2 L-1+2 n$.

Using powers of the matrices,

$$
H_{n l}^{m}:=\sum H_{n k_{1}} H_{k_{1} k_{2}} \cdots H_{k_{m}}
$$

and $G_{n l}$ the basis function can be represented as finite linear combinations of finer resolution scaling functions

$$
\begin{gathered}
s_{n}^{k}=\sum_{l} H_{n l}^{m} s_{l}^{k+m}, \\
w_{n}^{k}=\sum_{l t} H_{n t}^{m-1} G_{t l} s_{l}^{k+m},
\end{gathered}
$$

where the sums in (150) and (151) are finite. Using these identities, all of the integrals can be reduced to finite linear combinations of integrals involving a pair of scaling 
functions, $s_{n}^{k}(x)=2^{k / 2} s\left(2^{k} x-n\right)$, on a common fine scale, $2^{-k}$.

What remains is linear combinations of products of integrals of the form

$$
\begin{aligned}
& \int s_{m}^{k}(x) \epsilon\left(x^{-}-y^{-}\right) s_{n}^{k}\left(y^{-}\right) d x^{-} d y^{-} \\
& =\int 2^{k / 2} s\left(2^{k} x^{-}-m\right) \epsilon\left(x^{-}-y^{-}\right) 2^{k / 2} s\left(2^{k} y^{-}-n\right) d x^{-} d y^{-} .
\end{aligned}
$$

Changing variables

$$
y^{-\prime}=2^{k} y^{-}-n, \quad x^{-\prime}=2^{k} x^{-}-n,
$$

and noting

$$
\epsilon\left(x^{-}-y^{-}\right)=\epsilon\left(2^{k} x^{-}-2^{k} y^{-}\right),
$$

this becomes

$$
\begin{aligned}
& \int 2^{-k} s\left(x^{\prime-}-m\right) \epsilon\left(x^{\prime-}-y^{--}\right) s\left(y^{\prime-}-n\right) d x^{\prime-} d y^{\prime-}= \\
& \int 2^{-k} s\left(x^{\prime-}+n-m\right) \epsilon\left(x^{\prime-}-y^{\prime-}\right) s\left(y^{\prime-}\right) d x^{\prime-} d y^{\prime-}=2^{-k} I[n-m],
\end{aligned}
$$

where

$$
I[n]:=\int s\left(x^{-}+n\right) \epsilon\left(x^{-}-y^{-}\right) s\left(y^{-}\right) d x^{-} d y^{-} .
$$

$I[n]$ can be expressed as a difference of two integrals,

$$
I[n]=\int s\left(x^{-}+n\right)\left[\int_{-\infty}^{x^{-}} s\left(y^{-}\right)-\int_{x^{-}}^{\infty} s\left(y^{-}\right)\right] d x^{-} d y^{-},
$$

while the normalization condition (107) gives

$$
\int s\left(x^{-}+n\right)\left[\int_{-\infty}^{x^{-}} s\left(y^{-}\right)+\int_{x^{-}}^{\infty} s\left(y^{-}\right)\right] d x^{-} d y^{-}=1 .
$$

Adding (158) and (159) gives

$$
I[n]=2 \int s\left(x^{-}+n\right) \int_{-\infty}^{x^{-}} s\left(y^{-}\right) d x^{-} d y^{-}-1 .
$$

If the support of $s\left(x^{-}+n\right)$ is to the right of the support of $s\left(y^{-}\right)$, the integral is 1 while if the support of $s\left(x^{-}+n\right)$ is to the left of the support of $s\left(y^{-}\right)$the integral is -1 . Thus, for the $L=3$ basis functions,

$$
I[n]= \begin{cases}1 & n \leq=-5 \\ I[n] & -4 \leq n \leq 4 . \\ -1 & n \geq 5\end{cases}
$$

The $I[n]$ for $n \in[-4,4]$ are related by the renormalization group equations

$$
\begin{gathered}
I[n]=\int s\left(x^{-}+n\right) \epsilon\left(x^{-}-y^{-}\right) s\left(y^{-}\right) d x^{-} d y^{-}=, \\
2 \sum h_{l} h_{k} \int s\left(2 x^{-}+2 n-l\right) \epsilon\left(x^{-}-y^{-}\right) s\left(2 y^{-}-k\right) d x^{-} d y^{-}=, \\
\frac{1}{2} \sum h_{l} h_{k} \int s\left(2 x^{-}+2 n-l\right) \epsilon\left(2 x^{-}-2 y^{-}\right) s\left(2 y^{-}-k\right) 2 d x^{-} 2 d y^{-}=, \\
\frac{1}{2} \sum h_{l} h_{k} \int s\left(x^{-}+2 n-l\right) \epsilon\left(x^{-}-y^{-}\right) s\left(y^{-}-k\right) d x^{-} d y^{-}=, \\
\frac{1}{2} \sum h_{l} h_{k} \int s\left(x^{-}+2 n-l+k\right) \epsilon\left(x^{-}-y^{-}\right) s\left(y^{-}\right) d x^{-} d y^{-}=, \\
\frac{1}{2} \sum h_{l} h_{k} I[2 n+k-l]=\frac{1}{2} \sum h_{m+l-2 n} h_{l} I[m]=\frac{1}{4} \sum a_{m-2 n} I[m],
\end{gathered}
$$

where

$$
a_{n}:=2 \sum_{l=0}^{5} h_{l} h_{l+n} \quad-5 \leq n \leq 5 .
$$

The numbers $a_{n}$ will appear again. The $a_{n}$ are rational numbers [56-58]. For $\mathrm{L}=3$, the nonzero $a_{n}$ are

$$
a_{0}=2 \quad a_{1}=a_{-1}=\frac{75}{64} \quad a_{3}=a_{-3}=-\frac{25}{128} \quad a_{5}=a_{-5}=\frac{3}{128} .
$$


The $9 \times 9$ matrix $A_{m n}:=a_{n-2 m}(-4 \leq m, n \leq 4)$ has the following rational eigenvalues $\lambda=2,1, \frac{1}{2}, \frac{1}{4}, \pm \frac{1}{8}, \frac{1}{16}, \frac{9}{32}$, $-\frac{9}{64}$, so it is invertible.

The nontrivial $I[n]$ are solutions of the linear system,

$$
\sum_{n=-4}^{4} A_{m n} I[n]=d_{m}
$$

where

$$
d_{m}=a_{5-2 m}-a_{-5-2 m} .
$$

The solution of (170) is

$$
I[n]=\left(\begin{array}{rl}
-3.34201389 e+00, & n=-4 \\
8.33333333 e+00, & n=-3 \\
-1.79796007 e+01, & n=-2 \\
1.94444444 e+01, & n=-1 \\
0.00000000 e-00, & n=0 \\
-1.94444444 e+01, & n=1 \\
1.79796007 e+01, & n=2 \\
-8.33333333 e+00, & n=3 \\
3.34201389 e+00, & n=4
\end{array}\right) .
$$

While (172) is a numerical solution, the exact solution is rational since both $A_{m n}$ and $d_{n}$ are rational.

This solution, along with (161), can be used to construct the commutator of any of the discrete field operators using (145)-(155).

The general structure of the commutators is

$$
\begin{aligned}
{\left[\phi_{\mathbf{m}}(0), \phi_{\mathbf{n}}(0)\right] } & =C_{\mathbf{m}, \mathbf{n}} \\
& =(\text { scale factors }) \times(\text { powers of } \mathrm{H}, \mathrm{G}) \times I[n] .
\end{aligned}
$$

Note that while this commutator looks very nonlocal, if the scaling functions in (144) are replaced by wavelets with supports that are sufficiently separated, the integrals vanish because the moments of wavelets vanish. This will also be true of linear combinations of scaling functions that represent functions that vanish at $p^{+}=0$.

\section{POINCARÉ GENERATORS}

The other quantity needed to formulate the dynamics is an expression for $P^{-}$or one of the other dynamical Poincaré generators expressed in terms of operators in the irreducible algebra. Since the generators are conserved Noether charges, they are independent of $x^{+}$, so the generators can be expressed in terms of fields on the light front. The discrete representations of the generators can be constructed by replacing the fields on the light front by the discrete representation (124), (127) of the fields. The integrals over the light front become integrals over products of basis functions and their derivatives. This section discusses the computation of these integrals using renormalization group methods.

A scalar $\phi^{4}(x)$ theory is used for the purpose of illustration. In this case, the problem is to express all of the generators as linear combinations of products of discrete fields.

The construction of the Poincare generators from Noether's theorem was given in Sec. IV. Using the discrete representation of fields, the light-front Poincare generators (84)-(90) have the following forms:

$$
P^{+}=\sum_{\mathbf{m n}}: \phi_{\mathbf{m}}(0) \phi_{\mathbf{n}}(0): P_{\mathbf{m}, \mathbf{n}}^{+},
$$

where

$$
\begin{aligned}
P_{\mathbf{m}, \mathbf{n}}^{+} & :=2 \int d x^{-} d^{2} x_{\perp} \partial_{-} \xi_{\mathbf{m}}(\tilde{\mathbf{x}}) \partial_{-} \xi_{\mathbf{n}}(\tilde{\mathbf{x}}) \\
P^{i} & =\sum_{\mathbf{m n}}: \phi_{\mathbf{m}}(0) \phi_{\mathbf{n}}(0): P_{\mathbf{m}, \mathbf{n}}^{i}
\end{aligned}
$$

where

$$
\begin{gathered}
P_{\mathbf{m}, \mathbf{n}}^{i}:=-\int d x^{-} d^{2} x_{\perp} \partial_{-} \xi_{\mathbf{n}}(\tilde{\mathbf{x}}) \partial_{i} \xi_{\mathbf{m}}(\tilde{\mathbf{x}}), \\
P^{-}=\sum_{\mathbf{m n}}: \phi_{\mathbf{m}}(0) \phi_{\mathbf{n}}(0): P_{\mathbf{m}, \mathbf{n}}^{-}+\sum_{\mathbf{n}_{1} \mathbf{n}_{2} \mathbf{n}_{4} \mathbf{n}_{4}}: \phi_{\mathbf{n}_{1}}(0) \phi_{\mathbf{n}_{2}}(0) \phi_{\mathbf{n}_{3}}(0) \phi_{\mathbf{n}_{4}}(0): P_{\mathbf{n}_{1}, \mathbf{n}_{2}, \mathbf{n}_{3}, \mathbf{n}_{4}}^{-},
\end{gathered}
$$

where

$$
P_{\mathbf{m}, \mathbf{n}}^{-}:=\int d x^{-} d^{2} x_{\perp}\left(\frac{1}{2} \nabla_{\perp} \xi_{\mathbf{m}}(\tilde{\mathbf{x}}) \cdot \nabla_{\perp} \xi_{\mathbf{n}}(\tilde{\mathbf{x}})+\frac{1}{2} m^{2} \xi_{\mathbf{m}}(\tilde{\mathbf{x}}) \xi_{\mathbf{n}}(\tilde{\mathbf{x}})\right)
$$

and 


$$
\begin{gathered}
P_{\mathbf{n}_{1}, \mathbf{n}_{2}, \mathbf{n}_{3}, \mathbf{n}_{4}}^{-}:=\lambda \int d x^{-} d^{2} x_{\perp} \xi_{\mathbf{n}_{1}}(\tilde{\mathbf{x}}) \xi_{\mathbf{n}_{2}}(\tilde{\mathbf{x}}) \xi_{\mathbf{n}_{3}}(\tilde{\mathbf{x}}) \xi_{\mathbf{n}_{4}}(\tilde{\mathbf{x}}), \\
K^{3}=\sum_{\mathbf{m n}}: \phi_{\mathbf{m}}(0) \phi_{\mathbf{n}}(0): K_{\mathbf{m}, \mathbf{n}}^{3}+\sum_{\mathbf{n}_{1} \mathbf{n}_{2} \mathbf{n}_{4} \mathbf{n}_{4}}: \phi_{\mathbf{n}_{1}}(0) \phi_{\mathbf{n}_{2}}(0) \phi_{\mathbf{n}_{3}}(0) \phi_{\mathbf{n}_{4}}(0): K_{\mathbf{n}_{1}, \mathbf{n}_{2}, \mathbf{n}_{3}, \mathbf{n}_{4}}^{3},
\end{gathered}
$$

where

$$
K_{\mathbf{m}, \mathbf{n}}^{3}:=\int d x^{-} d^{2}\left(2 x_{\perp} x^{-} \partial_{-} \xi_{\mathbf{m}}(\tilde{\mathbf{x}}) \partial_{-} \xi_{\mathbf{n}}(\tilde{\mathbf{x}})-\frac{1}{2} x^{+} \boldsymbol{\nabla}_{\perp} \xi_{\mathbf{m}}(\tilde{\mathbf{x}}) \cdot \nabla_{\perp} \xi_{\mathbf{n}}(\tilde{\mathbf{x}})-\frac{1}{2} m^{2} x^{+} \xi_{\mathbf{m}}(\tilde{\mathbf{x}}) \xi_{\mathbf{n}}(\tilde{\mathbf{x}})\right)
$$

and

$$
K_{\mathbf{n}_{1}, \mathbf{n}_{2}, \mathbf{n}_{3}, \mathbf{n}_{4}}^{3}:=-\lambda \int d x^{-} d^{2} x_{\perp} x^{+} \xi_{\mathbf{n}_{1}}(\tilde{\mathbf{x}}) \xi_{\mathbf{n}_{2}}(\tilde{\mathbf{x}}) \xi_{\mathbf{n}_{3}}(\tilde{\mathbf{x}}) \xi_{\mathbf{n}_{4}}(\tilde{\mathbf{x}})
$$

Setting $x^{+}=0$, this becomes

$$
K_{\mathbf{m}, \mathbf{n}}^{3} \rightarrow 2 \int d x^{-} d^{2} x_{\perp} x^{-} \partial_{-} \xi_{\mathbf{m}}(\tilde{\mathbf{x}}) \partial_{-} \xi_{\mathbf{n}}(\tilde{\mathbf{x}}) ; \quad K_{\mathbf{n}_{1}, \mathbf{n}_{2}, \mathbf{n}_{3}, \mathbf{n}_{4}}^{3} \rightarrow 0 .
$$

For the remaining generators,

$$
E^{1}=\sum_{\mathbf{m n}}: \phi_{\mathbf{m}}(0) \phi_{\mathbf{n}}(0): E_{\mathbf{m}, \mathbf{n}}^{1}
$$

where

$$
\begin{gathered}
E_{\mathbf{m}, \mathbf{n}}^{1}:=\int d x^{-} d^{2} x_{\perp}\left(2 x^{1} \partial_{-} \xi_{\mathbf{m}}(\tilde{\mathbf{x}}) \partial_{-} \xi_{\mathbf{n}}(\tilde{\mathbf{x}})+\partial_{-} \xi_{\mathbf{m}}(\tilde{\mathbf{x}}) \partial_{1} \xi_{\mathbf{n}}(\tilde{\mathbf{x}}) x^{+}\right) \rightarrow 2 \int x^{1} \partial_{-} \xi_{\mathbf{m}}(\tilde{\mathbf{x}}) \partial_{-} \xi_{\mathbf{n}}(\tilde{\mathbf{x}}) \\
E^{2}=\sum_{\mathbf{m} \mathbf{n}}: \phi_{\mathbf{m}}(0) \phi_{\mathbf{n}}(0): E_{\mathbf{m}, \mathbf{n}}^{2}
\end{gathered}
$$

where

$$
\begin{gathered}
E_{\mathbf{m}, \mathbf{n}}^{2}:=\int d x^{-} d^{2} x_{\perp}\left(2 x^{2} \partial_{-} \xi_{\mathbf{m}}(\tilde{\mathbf{x}}) \partial_{-} \xi_{\mathbf{n}}(\tilde{\mathbf{x}})+\partial_{-} \xi_{\mathbf{m}}(\tilde{\mathbf{x}}) \partial_{2} \xi_{\mathbf{n}}(\tilde{\mathbf{x}}) x^{+}\right) \rightarrow 2 \int d x^{-} d^{2} x_{\perp} x^{2} \partial_{-} \xi_{\mathbf{m}}(\tilde{\mathbf{x}}) \partial_{-} \xi_{\mathbf{n}}(\tilde{\mathbf{x}}), \\
F^{1}=\sum_{\mathbf{m n}}: \phi_{\mathbf{m}}(0) \phi_{\mathbf{n}}(0): F_{\mathbf{m}, \mathbf{n}}^{1}+\sum_{\mathbf{n}_{1} \mathbf{n}_{2} \mathbf{n}_{3} \mathbf{n}_{4}}: \phi_{\mathbf{n}_{1}}(0) \phi_{\mathbf{n}_{2}}(0) \phi_{\mathbf{n}_{3}}(0) \phi_{\mathbf{n}_{4}}(0): F_{\mathbf{n}_{1}, \mathbf{n}_{2}, \mathbf{n}_{3}, \mathbf{n}_{4}}^{1},
\end{gathered}
$$

where

$$
F_{\mathbf{m}, \mathbf{n}}^{1}:=\int d x^{-} d^{2} x_{\perp}\left(\frac{1}{2} x^{1} \nabla_{\perp} \xi_{\mathbf{k}}(x) \cdot \nabla_{\perp} \xi_{\mathbf{l}}(x)+\frac{1}{2} x^{1} m^{2} \xi_{\mathbf{k}}(\tilde{\mathbf{x}}) \xi_{\mathbf{l}}(\tilde{\mathbf{x}})+x^{-} \partial_{-} \xi_{\mathbf{k}}(\tilde{\mathbf{x}}) \partial_{1} \xi_{\mathbf{l}}(\tilde{\mathbf{x}})\right)
$$

and

$$
\begin{gathered}
F_{\mathbf{n}_{1}, \mathbf{n}_{2}, \mathbf{n}_{3}, \mathbf{n}_{4}}^{1}:=\lambda \int d x^{-} d^{2} x_{\perp} x^{1} \xi_{\mathbf{n}_{1}}(\tilde{\mathbf{x}}) \xi_{\mathbf{n}_{2}}(\tilde{\mathbf{x}}) \xi_{\mathbf{n}_{3}}(\tilde{\mathbf{x}}) \xi_{\mathbf{n}_{4}}(\tilde{\mathbf{x}}), \\
F^{2}=\sum_{\mathbf{m n}}: \phi_{\mathbf{m}}(0) \phi_{\mathbf{n}}(0): F_{\mathbf{m}, \mathbf{n}}^{2}+\sum_{\mathbf{n}_{1} \mathbf{n}_{2} \mathbf{n}_{4} \mathbf{n}_{4}}: \phi_{\mathbf{n}_{1}}(0) \phi_{\mathbf{n}_{2}}(0) \phi_{\mathbf{n}_{3}}(0) \phi_{\mathbf{n}_{4}}(0): F_{\mathbf{n}_{1}, \mathbf{n}_{2}, \mathbf{n}_{3}, \mathbf{n}_{4}}^{2},
\end{gathered}
$$

where 


$$
F_{\mathbf{m}, \mathbf{n}}^{2}:=\int d x^{-} d^{2} x_{\perp}\left(\frac{1}{2} x^{2} \nabla_{\perp} \xi_{\mathbf{k}}(x) \cdot \nabla_{\perp} \xi_{\mathbf{l}}(x)+\frac{1}{2} x^{2} m^{2} \xi_{\mathbf{k}}(\tilde{\mathbf{x}}) \xi_{\mathbf{l}}(\tilde{\mathbf{x}})+x^{-} \partial_{-} \xi_{\mathbf{k}}(\tilde{\mathbf{x}}) \partial_{2} \xi_{\mathbf{l}}(\tilde{\mathbf{x}})\right)
$$

and

$$
F_{\mathbf{n}_{1}, \mathbf{n}_{2}, \mathbf{n}_{3}, \mathbf{n}_{4}}^{2}:=\lambda \int d x^{-} d^{2} x_{\perp} x^{2} \xi_{\mathbf{n}_{1}}(\tilde{\mathbf{x}}) \xi_{\mathbf{n}_{2}}(\tilde{\mathbf{x}}) \xi_{\mathbf{n}_{3}}(\tilde{\mathbf{x}}) \xi_{\mathbf{n}_{4}}(\tilde{\mathbf{x}})
$$

All of these operators have the structure of linear combinations of normal products of discrete fields evaluated at $x^{+}=0$ times constant coefficients, $P_{\mathbf{n}_{1}, \mathbf{n}_{2}}^{+}, P_{\mathbf{n}_{1}, \mathbf{n}_{2}}^{i}, P_{\mathbf{n}_{1}, \mathbf{n}_{2}}^{-}, P_{\mathbf{n}_{1}, \mathbf{n}_{2}, \mathbf{n}_{3}, \mathbf{n}_{4}}^{-}, K_{\mathbf{n}_{1}, \mathbf{n}_{2}}^{3}, J_{\mathbf{n}_{1}, \mathbf{n}_{2}}^{3}, E_{\mathbf{n}_{1}, \mathbf{n}_{2}}^{i}, F_{\mathbf{n}_{1}, \mathbf{n}_{2}}^{i}, F_{\mathbf{n}_{1}, \mathbf{n}_{2}, \mathbf{n}_{3}, \mathbf{n}_{4}}^{i}$, which are integrals involving products of basis functions and their derivatives. The three-dimensional integrals that need to be evaluated to compute these coefficients are products of 3 one-dimensional integrals that have one of the following eight forms:

$$
\begin{gathered}
\int d x \xi_{m}(x) \xi_{n}(x) \quad \int d x \partial_{x} \xi_{m}(x) \xi_{n}(x) \quad \int d x \partial_{x} \xi_{m}(x) \partial_{x} \xi_{n}(x), \\
\int d x x \xi_{m}(x) \xi_{n}(x) \quad \int d x x \partial_{x} \xi_{m}(x) \xi_{n}(x) \quad \int d x x \partial_{x} \xi_{m}(x) \partial_{x} \xi_{n}(x), \\
\int d x \xi_{n_{1}}(x) \xi_{n_{2}}(x) \xi_{n_{3}}(x) \xi_{n_{4}}(x) \quad \int d x x \xi_{n_{1}}(x) \xi_{n_{2}}(x) \xi_{n_{3}}(x) \xi_{n_{4}}(x) .
\end{gathered}
$$

In what follows, it is shown how all of these integrals can be computed using the renormalization group equation (104).

The integrals (195)-(197) are products of basis functions which may be scaling functions with scale $2^{-k}$ or wavelets of scale $2^{-k-l}$ for $l \geq 0$. The same methods that were used in the computation of the commutator function, (145)(151), can be used to express the integrals (195)-(197) as linear combinations of integrals involving scaling functions on a common scale fine scale, $2^{-l}$.

$$
\begin{gathered}
\int d x s_{m}^{l}(x) s_{n}^{l}(x)=\delta_{m n} \\
\int d x \partial_{x} s_{m}^{l}(x) s_{n}^{l}(x)=2^{l} \int d x s^{\prime}(x) s_{n-m}(x) \\
\int d x \partial_{x} s_{m}^{l}(x) \partial_{x} s_{n}^{l}(x)=2^{2 l} \int d x s^{\prime}(x) s_{n-m}^{\prime}(x) \\
\int d x s_{n_{1}}^{l}(x) s_{n_{2}}^{l}(x) s_{n_{3}}^{l}(x) s_{n_{4}}^{l}(x)=2^{l} \int d x s(x) s_{n_{2}-n_{1}}(x) s_{n_{3}-n_{1}}(x) s_{n_{4}-n_{1}}(x) \\
\int d x x s_{m}^{l}(x) s_{n}^{l}(x)=2^{-l}\left(\int d x x s(x) s_{n-m}(x)+m \delta_{m, n}\right) \\
\int d x x \partial_{x} s_{m}^{l}(x) s_{n}^{l}(x)=\int d x(x+m) s^{\prime}(x) s_{n-m}(x) \\
\int d x x \partial_{x} s_{m}^{l}(x) \partial_{x} s_{n}^{l}(x)=2^{l} \int d x(x+m) s^{\prime}(x) s_{n-m}^{\prime}(x) \\
\int d x x s_{n_{1}}^{l}(x) s_{n_{2}}^{l}(x) s_{n_{3}}^{l}(x) s_{n_{4}}^{l}(x)=\int d x\left(x+n_{1}\right) s(x) s_{n_{2}-n_{1}}(x) s_{n_{3}-n_{1}}(x) s_{n_{4}-n_{1}}(x) .
\end{gathered}
$$


These identities express all of the integrals involving scale $2^{-l}$ scaling functions in terms of related integrals involving the $s_{n}(x)$. The compact support of the functions $s_{n}(x)$ means these integrals are identically zero unless the indices and the absolute values of their differences are less than or equal to $2 L-2$ which is 4 for $L=3$.

The integrals of the right side of (199)-(205) are the following integrals:

$$
\begin{gathered}
\delta_{m n}=\int d x s_{m}(x) s_{n}(x) \quad m=n, \\
D_{1}[m]:=\int d x \frac{d s}{d x}(x) s_{m}(x) \quad-4 \leq m \leq 4, \\
D_{2}[m]:=\int d x \frac{d s}{d x}(x) \frac{d s_{m}}{d x}(x) \quad-4 \leq m \leq 4, \\
\Gamma_{4}[m][n][k]:=\int d x s(x) s_{m}(x) s_{n}(x) s_{k}(x) \\
-4 \leq m, n, k, m-n, m-k, k-n \leq 4, \\
X[m]:=\int d x x s(x) s_{m}(x) \quad-4 \leq m \leq 4, \\
X_{1}[m]:=\int d x x \frac{d s}{d x}(x) s_{m}(x) \quad-4 \leq m \leq 4, \\
X_{2}[m]:=\int d x x \frac{d s}{d x}(x) \frac{d s_{m}}{d x}(x) \quad-4 \leq m \leq 4,
\end{gathered}
$$

$\Gamma_{4}[m][n][k]:=\sum_{l, l_{m} l_{n}, l_{k}=0}^{5} 2 h_{l} h_{l_{m}} h_{l_{n}} h_{l_{k}} \Gamma_{4}\left[2 m+l_{m}-l\right]\left[2 n+l_{n}-l\right]\left[2 k+l_{k}-l\right]=\sum_{m^{\prime} m^{\prime} k^{\prime}} A_{4}\left(m, n, k ; m^{\prime}, n^{\prime}, k^{\prime}\right) \Gamma_{4}\left[m^{\prime}\right]\left[n^{\prime}\right]\left[k^{\prime}\right]$,

where

$A_{4}\left(m, n, k ; m^{\prime}, n^{\prime}, k^{\prime}\right):=\sum_{l} 2 h_{l} h_{m^{\prime}-2 m+l} h_{n^{\prime}-2 n+l} h_{k^{\prime}-2 k+l}$.

The relations involving $X[n], X 1[n], X 2[n]$ and $\Gamma_{4 x}[m][n][k]$ have inhomogeneous parts,

$$
\begin{array}{r}
X[n]=\frac{1}{4} \sum_{m=-4}^{4} A_{n m} X[m]+\frac{1}{2} \sum_{l} l h_{l} h_{l-2 n} \\
X_{1}[n]=\frac{1}{2} \sum_{m=-4}^{4} A_{n m} X_{1}[m]+\sum_{l} l h_{l} h_{l-2 n+m} D_{1}[m] \\
X_{2}[n]=\sum_{m=-4}^{4} A_{n m} X_{2}[m]+2 \sum_{l} l h_{l} h_{l-2 n+m} D_{2}[m]
\end{array}
$$

$$
\begin{aligned}
\Gamma_{4 x}[m][n][k] & :=\int d x x s(x) s_{m}(x) s_{n}(x) s_{k}(x) \\
-4 & \leq m, n, k, m-n, m-k, k-n \leq 4 .
\end{aligned}
$$

The renormalization group equation in the form

$$
s(x-n)=\sum_{l=0}^{5} h_{l} \sqrt{2} s(2 x-2 n-l)
$$

and a variable change $x \rightarrow x^{\prime}=2 x$ lead to the following linear equations relating the nonzero values of these integrals:

$$
\begin{array}{r}
D_{1}[n]=\sum_{m=-4}^{4} a_{m-2 n} D_{1}[m]=\sum_{m=-4}^{4} A_{n m} D_{1}[m], \\
D_{2}[n]=2 \sum_{m=-4}^{4} a_{m-2 n} D_{2}[m]=2 \sum_{m=-4}^{4} A_{n m} D_{2}[m],
\end{array}
$$

where $a_{m}$

$$
a_{m}:=2 \sum_{k=0}^{5} h_{k+m} h_{k} \quad-5 \leq m \leq 5
$$

is the same quantity (168) and (169) that appeared in the computation of the commutator function. A similar quantity appears in the homogeneous equations relating the nonzero $\Gamma_{4}[m][n][k]$ 's,$$
\Gamma_{4 x}[m][n][k]:=\frac{1}{2} \sum_{m^{\prime} n^{\prime} k^{\prime}} A_{4}\left(m, n, k ; m^{\prime}, n^{\prime}, k^{\prime}\right) \Gamma_{4 x}\left[m^{\prime}\right]\left[n^{\prime}\right]\left[k^{\prime}\right]-,
$$$$
\sum_{m^{\prime} n^{\prime} k^{\prime}}\left(\sum_{l} h_{l} h_{m^{\prime}-2 m+l} h_{n^{\prime}-2 n+l} h_{k^{\prime}-2 k+l} l\right) \Gamma_{4}\left[m^{\prime}\right]\left[n^{\prime}\right]\left[k^{\prime}\right] .
$$

Since the $9 \times 9$ matrix $A_{m n}:=a_{n-2 m}(-4 \leq m, n \leq 4)$ has eigenvalues $\lambda=2,1, \frac{1}{2}, \frac{1}{4}, \pm \frac{1}{8}, \frac{1}{16}, \frac{9}{32},-\frac{9}{64}$, it follows that $D_{1}[n]$ and $D_{2}[n]$ are eigenvectors of $A_{m n}$ with eigenvalues 1 and $\frac{1}{2}$, respectively. The normalization is determined by the equations discussed below. Equation (218) similarly implies that $\Gamma_{4}[m][n][k]$ is an eigenvector with eigenvalue 1 of the matrix $A_{4}$ defined by the right-hand side of (218). The normalization of $\Gamma_{4}[m][n][k]$ is also discussed below. 
The matrix $\left(I-\frac{1}{4} A\right)$ in (220) is invertible, so (220) is a well-posed linear system for $X[n]$, while the matrices $\left(I-\frac{1}{2} A\right)$ and $(I-A)$ in (221) and (222) are singular. To solve them, the Moore-Penrose generalized inverse [59] is applied to the inhomogeneous terms to get specific solutions. These solutions are substituted back in the equations to ensure that the inhomogeneous terms are in the range of $\left(I-\frac{1}{2} A\right)$ and $(I-A)$, respectively, although this must be the case since the solutions can also be expressed as integrals. The general solutions of (221) and (222) can include arbitrary amounts of the solution of the homogeneous equations which are eigenstates of $A_{m n}$ with eigenvalues 2 and 1, respectively. The contribution from the homogeneous equation is determined by the normalization conditions below.

The normalization conditions are derived from the property that polynomials with degree less than $L$ can be pointwise represented as locally finite-linear combination of the $s_{n}(x)$. These expansions have the form

$$
\begin{gathered}
1=\sum s_{n}(x), \\
x=\sum(\langle x\rangle+n) s_{n}(x)=\langle x\rangle+\sum n s_{n}(x), \\
x^{2}=\sum(\langle x\rangle+n)^{2} s_{n}(x)=\langle x\rangle^{2}+2\langle x\rangle \sum n s_{n}(x) \\
+\sum n^{2} s_{n}(x),
\end{gathered}
$$

where

$$
\left\langle x^{n}\right\rangle:=\int s(x) x^{n} d x
$$

are moments of $s(x)$. Differentiating (226) and (227) gives

$$
\begin{gathered}
1=\sum n s_{n}^{\prime}(x), \\
x=\langle x\rangle+\frac{1}{2} \sum n^{2} s_{n}^{\prime}(x) .
\end{gathered}
$$

Multiplying (229) by $s(x)$ and integrating the result give

$$
\sum_{n=-4}^{4} n D_{1}[n]=-1
$$

Multiplying (230) by $s^{\prime}(x)$ and integrating give

$$
\sum_{n=-4}^{4} n^{2} D_{2}[n]=-2
$$

These conditions determine the normalization of the eigenvectors $D_{1}[n]$ and $D_{2}[n]$. Note that the moments do not appear in these normalization conditions, although all moments of $s(x)$ can be computed recursively using the renormalization group equation and the normalization condition (107). Using (229) in (211) and integrating by parts gives

$$
\sum_{n=-4}^{4} X_{1}[n]=-1
$$

Using (230) in (212) and integrating by parts gives

$$
\sum_{n=-4}^{4} n X_{2}[n]=-1 \text {. }
$$

These conditions determine the contribution of the solution of the homogeneous equations in the general solution.

The normalization conditions for $\Gamma_{4}[m][n][k]$ are obtained using the partition of unity property (225),

$$
\sum_{m=-4}^{4} \Gamma_{4}[m][n][k]=\Gamma_{3}[n][k] ; \quad \sum_{n=-4}^{4} \Gamma_{3}[n][k]=\delta_{k 0},
$$

$$
\sum_{m=-4}^{4} \Gamma_{4 x}[m][n][k]=\Gamma_{3 x}[n][k] ; \quad \sum_{n=-4}^{4} \Gamma_{3}[n][k]=X[k],
$$

where

$$
\begin{array}{ll}
\Gamma_{3}[m][n]:=\int d x x s(x) s_{m}(x) s_{n}(x) & -2 L+2 \leq m, n, m-n \leq 2 L-2, \\
\Gamma_{3 x}[m][n]:=\int d x x s(x) s_{m}(x) s_{n}(x) & -2 L+2 \leq m, n, m-n \leq 2 L-2,
\end{array}
$$

and $\Gamma_{3}[m][n]$ is a solution of the eigenvalue problem

$$
\Gamma_{3}[m][n]=\sum_{m^{\prime} n^{\prime}} a_{3}\left(m, n ; m^{\prime} n^{\prime}\right) \Gamma_{3}\left[m^{\prime}\right]\left[n^{\prime}\right]
$$

with normalization (235) and 


$$
a_{3}\left(m, n ; m^{\prime} n^{\prime}\right)=\sum_{l} h_{l} h_{m^{\prime}-2 m+l} h_{n^{\prime}-2 n+l}
$$

$\Gamma_{3 x}[m][n]$ satisfies

$$
\begin{gathered}
\Gamma_{3 x}[m][n]:=\sum_{m^{\prime} n^{\prime}} a_{3}\left(m, n ; m^{\prime} n^{\prime}\right) \Gamma_{3 x}\left[m^{\prime}\right]\left[n^{\prime}\right]-, \\
\sum_{m^{\prime} n^{\prime}}\left(\sum_{l}^{2 L-1} l h_{l} h_{m^{\prime}-2 m+l} h_{n^{\prime}-2 n+l}\right) \Gamma_{3}\left[m^{\prime}\right]\left[n^{\prime}\right],
\end{gathered}
$$

with the normalization constraint

$$
\sum_{n} \Gamma_{3 x}[m][n]=X[m]
$$

These finite linear systems can be solved for all of the integrals (195)-(197). The results for $D 1[n], D 2[n], X[n]$, $X 1[n], X 2[n]$ for $L=3$, which are needed to compute the constant coefficients for the free-field generators, are given below. The vector $\Gamma_{4}[m][n][k]$ of coefficients for the dynamical generators has too many components to display. They can be computed by finding the eigenvector with eigenvalue 1 of the $9^{3} \times 9^{3}$ matrix $a_{4}[m][n]\left[m^{\prime}\right]\left[n^{\prime}\right]$ with normalization given by (235). The normalization condition requires solving for the eigenvector with eigenvalues 1 of the $9^{2} \times 9^{2}$ matrix $a_{3}[m][n]\left[m^{\prime}\right]\left[n^{\prime}\right]$ using the normalization condition (235). Finally, $\Gamma_{4 x}[m][n][k]$ be computed by applying the Moore Penrose generalized inverse of $\left(I-a_{4}\right)$ to the inhomogeneous term in (223) and adding an amount of the solution of the eigenvalue problem $\left(2 I-a_{4}\right) X=0$ consistent with the normalization condition (236).

All the these quantities can alternatively computed by a direct quadrature; however, the fractal nature of the basis functions makes the renormalization group method discussed above preferable. The values of $D 1[n], D 2[n], X[n]$, $X 1[n]$, and $X 2[n]$ are given below.

$$
\left(\begin{array}{l}
D 1[-4]=\frac{1}{2920} \\
D 1[-3]=-\frac{16}{1095} \\
D 1[-2]=-\frac{53}{365} \\
D 1[-1]=\frac{272}{365} \\
D 1[0]=0.0 \\
D 1[1]=-\frac{272}{365} \\
D 1[2]=\frac{53}{365} \\
D 1[3]=-\frac{16}{1095} \\
D 1[4]=-\frac{1}{2920}
\end{array}\right)\left(\begin{array}{l}
D 2[-4]=-\frac{3}{560} \\
D 2[-3]=-\frac{4}{35} \\
D 2[-2]=\frac{92}{105} \\
D 2[-1]=-\frac{356}{105} \\
D 2[0]=\frac{295}{56} \\
D 2[1]=-\frac{356}{105} \\
D 2[2]=\frac{92}{105} \\
D 2[3]=-\frac{4}{35} \\
D 2[4]=-\frac{3}{560}
\end{array}\right)
$$

\section{TRUNCATIONS}

The value of the wavelet representation is that, while it is formally exact, it also admits natural volume and resolution truncations in the light-front hyperplane. Truncations define effective theories that are expected to be good approximations to the theory for reactions associated with a volume and energy scale corresponding to the volume and resolution of the truncations. The simplest truncation discards degrees of freedom smaller than some limiting fine resolution, $2^{-l}$, as well as degrees of freedom with support outside of some volume on the light front.
In this regard, it has similar properties to a lattice truncation. Unlike a lattice truncation, because the theory is formally exact, it is straightforward to systematically include corrections associated with finer resolution or larger volumes. Some other appealing features are that the truncated fields have a continuous space-time dependence and can be differentiated, so there is no need to use finite difference approximations. Finally, it is possible to take advantage of some of the advantages of the light-front quantization.

One problem that is common to lattice truncations of field theory is that truncations break symmetries. In the 
light-front case, truncations break the kinematic covariance. One consequence is that transforming the truncated field covariantly using (128) is not the same as transforming the truncated field using the matrix (130) and truncating the result. The difference between these two calculations is due to the discarded degrees of freedom, which should be small for a suitable truncation. This suggests that kinematic Lorentz transformations can be approximated by using (128) with the truncated fields. The vacuum of the formally exact theory is the trivial Fock vacuum if the interaction commutes with the kinematic subgroup. When the kinematic invariance is broken, the lowest mass eigenstate of the truncated $P^{-}$is not necessarily the Fock vacuum; however, the Fock vacuum should become the lowest mass state in the infinite-volume, zeroresolution limit. This suggests that using trivial Fock vacuum might still be a good approximation.

The basis discussed in this work is not the only possible basis choice and may not be the best option for treating the transverse degrees of freedom for fields in $3+1$ dimensions. In this work, the transverse degrees of freedom are expanded in products of multiscale basis functions of Cartesian coordinates, $x$ and $y$. Truncations of this basis break the rotational symmetry about the $z$ axis. An alternative is to expand the transverse degrees of freedom in a basis consisting of products of functions of the polar coordinates $r$ and $\theta$ where $x=r \cos (\theta)$ and $y=r \sin (\theta)$. The basis functions in the $\theta$ variable can be taken as the periodic functions, $\frac{1}{\sqrt{2 \pi}} e^{i n \theta}$. This choice maintains the rotational symmetry, but does not give a multiresolution treatment of the angle degree of freedom. A second option is to use the multiresolution basis in the angle variable on $[0,2 \pi]$ with periodic boundary conditions. In this case, the truncations will result in a discrete rotational symmetry that depends on the resolution. In both cases, the radial degree of freedom can be expanded in a multiresolution basis. The only difference is that the radial functions have support on $[0, \infty]$ rather than $[-\infty, \infty]$. This requires replacing the basis functions that have support at $r=0$ by linear combinations of these functions that satisfy the boundary conditions at the origin. The linear combinations in a subspace of a given resolution can be constructed so they are orthonormal on $[0, \infty]$, resulting in an orthonormal basis on that subspace; however, the modified basis functions near the origin in subspaces of different resolution are no longer orthogonal. This results in additional coupling of degrees of freedom on different scales near $r=0$. This is because the exact boundary conditions at $r=0$ involve functions of all resolutions.

\section{SUMMARY AND OUTLOOK}

This work introduced a multiresolution representation of quantum field theory on a light front. This is a formally exact representation of the field theory in terms of an infinite number of discrete degrees of freedom that are localized on the light front. Each degree of freedom is associated with a compact subset of the light front. These subsets cover the light front, and there are an infinite number of them in every open subset on the light front. This representation has the property that there are a finite number of these degrees of freedom associated with any finite volume and any given maximal resolution on the light front.

Each degree of freedom or mode is represented by a field on the light front integrated over a basis function of compact support on the light front. The discrete fields associated with a free-field theory are an irreducible set of operators on the free-field Fock space. For interacting theories with self-adjoint kinematically invariant interactions, the spectral condition on $P^{+}$implies that the interaction cannot change the Fock vacuum. This means dynamical operators like the Poincaré generators can be expressed as functions of this irreducible algebra of fields acting on the free field Fock space.

The Poincaré generators involve ill-defined products of fields at the same point, so the formal interactions are not well-defined self-adjoint operators on the Fock space. In the multiresolution representation, the ultraviolet singularities that arise from local operator products necessarily appear as nonconvergence of infinite sums of well-defined operator products. There are also infrared divergences that appear in products of scaling function modes even after the smearing. In the light-front case, the ultraviolet and infrared singularities are constrained by rotational covariance, so any strategy to nonperturbatively renormalize the theory must treat these problems together.

Computations necessarily involve both volume and resolution cutoffs, which result in a well-defined truncated theory with a finite number of degrees of freedom. As long as the interaction in the truncated theory vanishes at $p^{+}=0$, the interaction will leave the Fock vacuum unchanged. The variable $p^{+}=\hat{\mathbf{z}} \cdot \mathbf{p}+\sqrt{m^{2}+\mathbf{p}^{2}}$ approaches zero in the limit that $-\hat{\mathbf{z}} \cdot \mathbf{p} \rightarrow+\infty$, so it is an infinite momentum limit, which involves high-resolution degrees of freedom. Requiring that the interaction vanish at $p^{+}=0$ is a resolution cutoff. This can be realized by discarding products of scaling function modes in the interaction. These modes do not contribute to the operator product when it is integrated over functions with vanishing Fourier transforms at $p^{+}=0$.

Dynamical calculations evolve the fields to points off of the light front. This evolution can be performed by iterating the light-front Heisenberg field equations or by solving the light-front Schrodinger equation. Both cases involve discrete mathematics. Iterating the Heisenberg field equations results in a representation of the field as an expansion in normal products of discrete fields on the light front with $x^{+}$dependent coefficients. Because fields on the light front are irreducible, the different discrete field operators cannot all 
commute; however, the commutator can be calculated explicitly and analytically. Vacuum expectation values of product of fields can be computed by evaluating the solution of the Heisenberg equations in the Fock vacuum.

There are a number of problems involving free fields that can be used to try to understand the convergence of computational strategies in truncated theories. Free-field theories have the advantage that they can be solved exactly, so errors can be calculated by comparing exact computations to computations based on truncated theories. Among the problems of interest is how is Poincare invariance recovered as the resolution is increased in a truncated theory. Because the basis is local, this can be tested in a finite volume. Methods for performing this test in the corresponding wavelet representation of canonical field theory were discussed in [25]. These methods utilize the locally finite partition of unity property of the scaling functions in the expression for the generators in terms of the integrals over the energy-momentum and angular-momentum tensor densities. In the light-front case, free fields provide a laboratory to investigate the accuracy of kinematic Lorentz transformations in truncated theories. Another important problem is how efficiently can the multiresolution representation of the light-front Hamiltonian be block diagonalized by resolution. This was studied for the case of the corresponding wavelet representation of canonical field theory in [35]. One conclusion of that work is that both volume and resolution need to be increased simultaneously in order to converge to a sensible energy spectrum of the Hamiltonian (i.e., so it approaches a continuous spectrum that is unbounded above). In addition, it was found that convergence to a block diagonal form slowed as energy separation of the modes decreased. Another calculation that should be done is to compare the Wightman functions or commutator functions of the truncated theories to the exact quantities. The light-front representation has the advantage that these can be computed without solving for an approximate vacuum. Another interesting question is what is the contribution of the product of the infrared singular parts of the truncated fields to normal ordered products of free fields. Does the normal ordering remove these contributions?

The next class of problems of interest are $1+1$-dimensional solvable field theories. These are interesting because the dynamical equations in the multiresolution representation generate more complicated operators in the algebra of fields on the light front. Reference [15] used Daubechies' wavelets methods in a canonical representation of the field theory to treat the X-Y model and spontaneous symmetry breaking in the Landau Ginzburg model.

The real interest is to apply multiresolution methods to realistic theories in $3+1$ dimensions. These are computationally far more complex than problems involving free fields or problem in low dimensions. There are several kinds of problems of interest. These include bound state problems, scattering problems, studies of correlation functions, and extensions to gauge theories. While the discrete nature of the multiresolution representation has some computational advantages, they will not be of significant help for these complex problems, especially since the number of modes scale with dimension and number of particles. One of the advantages of multiresolution methods is that basis functions are self-similar. The result is that the coupling strength of the various modes differs by different powers of 2. A systematic investigation could help to identify the most dominant modes in a given application. This could be used to get a rough first approximation that can be improved perturbatively. One interesting property of the multiresolution representation of the theory is that it is both discrete and formally exact. In a formally exact theory, Haag-Ruelle scattering theory can be used to express scattering observables as strong limits. Of interest is to use the exact representation to develop an approximation algorithm for computing scattering observables in this discrete representation. This is not trivial, since the time limits will not converge if they are computed after truncation.

Bound state calculations could be computed by diagonalizing the mass operator on a subspace, similar to how this is done using basis light-front quantization [47]. Variational methods could prove useful in this regard.

For gauge theories, the exact representation of the field theory in terms of a countable number of discrete fields with different resolutions suggest that a similar construction could be performed in using of gauge-invariant degrees of freedom. To understand how this might work, imagine a set of gauge-invariant Wilson placquets with a given lattice spacing on a light front. The expectation is that on the light front these form an irreducible algebra of operators of a given resolution. Decreasing the lattice spacing by a factor of 2 results in a new algebra that is an irreducible set of operators for the increased resolution. The coarse-scale algebra should be a subalgebra of the fine-scale algebra. In the same way, that scaling functions on a file scale can be expressed as wavelet and scaling functions on a coarse scale, it can be anticipated that there is something like a wavelet transform that generates the fine-scale algebra in terms of generators for the coarse-scale algebra and independent gauge-invariant operators that generate the degrees of freedom in the fine-scale algebra that are not in the coarse-scale algebra. As in the wavelet case, this could be repeated on every scale, leading to a countable set of independent operators that can generate placquets on all scales. This should result in an irreducible set of gaugeinvariant operators on the light front, with a formally trivial vacuum. While this construction is far from trivial, having a formally exact representation of gauge theories in terms of local gauge-invariant variables is a desirable goal. 
Another class of applications where the wavelet representation may be useful is in quantum computing. The fundamental property is that the local nature of the interactions involving different discrete modes means that transfer matrices for small time steps can be expressed as simple quantum circuits. Some comments on using wavelet discretized fields in quantum computing appear in
Refs. [29,33]. The advantage in the light-front case is the trivial nature of the vacuum.

\section{ACKNOWLEDGMENTS}

The author would like to acknowledge generous support from the U.S. Department of Energy, Office of Science, Nuclear Theory Program, Grant No. DE-SC16457.
[1] E. P. Wigner, Ann. Math. 40, 149 (1939).

[2] P. A. M. Dirac, Rev. Mod. Phys. 21, 392 (1949).

[3] S. Fubini and G. Furlan, Physics 1, 229 (1965).

[4] S. Weinberg, Phys. Rev. 150, 1313 (1966).

[5] L. Susskind, Phys. Rev. 165, 1535 (1968).

[6] K. Bardakci and M. B. Halpern, Phys. Rev. 176, 1686 (1968).

[7] S. J. Chang and S. Ma, Phys. Rev. 180, 1506 (1969).

[8] D. E. Soper and J. B. Kogut, Phys. Rev. D 1, 2901 (1970).

[9] H. Leutwyler, J. R. Klauder, and L. Streit, Nuovo Cimento A 66, 536 (1970).

[10] S. Schlieder and E. Seiler, Commun. Math. Phys. 25, 62 (1972).

[11] S.-J. Chang, R. G. Root, and T.-M. Yan, Phys. Rev. D 7, 1133 (1973).

[12] F. Coester, Prog. Part. Nucl. Phys. 29, 1 (1992).

[13] K. G. Wilson, T. S. Walhout, A. Harindranath, W.-M. Zhang, R. J. Perry, and S. D. Glazek, Phys. Rev. D 49, 6720 (1994).

[14] S. Brodsky, H.-C. Pauli, and S. Pinsky, Phys. Rep. 301, 299 (1998).

[15] C. Best and A. Schaefer, arXiv:hep-lat/9402012.

[16] P. Federbush, Prog. Theor. Phys. 94, 1135 (1995).

[17] I. G. Halliday and P. Suranyi, Nucl. Phys. B436, 414 (1995).

[18] G. Battle, Wavelets and Renormalization, Series in Approximations and Decompositions Vol. 10 (World Scientific, Singapore, 1999).

[19] C. Best, Nucl. Phys. Proc. Suppl. 83, 848 (2000).

[20] A. E. Ismail, G. C. Rutledge, and G. Stephanopoulos, J. Chem. Phys. 118, 4414 (2003).

[21] A. E. Ismail, G. C. Rutledge, and G. Stephanopoulos, J. Chem. Phys. 118, 4424 (2003).

[22] M. V. Altaisky, SIGMA 3, 105 (2007).

[23] S. Albeverio and M. V. Altaisky, arXiv:0901.2806v2.

[24] M. V. Altaisky, Phys. Rev. D 81, 125003 (2010).

[25] F. Bulut and W. N. Polyzou, Phys. Rev. D 87, 116011 (2013).

[26] M. V. Altaisky and N.E. Kaputkina, Phys. Rev. D 88, 025015 (2013).

[27] M. V. Altaisky and N. E. Kaputkina, Izv. Vuz. Fiz. 10, 68 (2012) [Russ. Phys. J. 55, 1177 (2013)].

[28] W. N. Polyzou and F. Bulut, Few-Body Syst. 55, 561 (2014).
[29] G. K. Brennen, P. Rohde, B. C. Sanders, and S. Singh, Phys. Rev. A 92, 032315 (2015).

[30] M. V. Altaisky, arXiv:1604.03431.

[31] M. V. Altaisky and N. E. Kaputkina, Int. J. Theor. Phys. 55, 2805 (2016).

[32] M. V. Altaisky, Phys. Rev. D 93, 105043 (2016).

[33] G. Evenbly and S. R. White, Phys. Rev. Lett. 116, 140403 (2016).

[34] H. Neuberger, arXiv:1707.09623v1.

[35] T. Michlin, W. N. Polyzou, and F. Bulut, Phys. Rev. D 95, 094501 (2017).

[36] M. V. Altaisky, Phys. At. Nucl. 81, 786 (2018).

[37] I. Daubechies, Commun. Pure Appl. Math. 41, 909 (1988).

[38] I. Daubechies, Ten Lectures on Wavelets, CBMS-NSF Regional Conference Series in Applied Mathematics (SIAM, Philadelphia, 1992).

[39] G. Kaiser, A Friendly Guide to Wavelets (Birkhäuser, Boston, 1994).

[40] H. L. Resnikoff and R. O. Wells, Jr., Wavelet Analysis (Springer, New York, 1998).

[41] O. Bratteli and P. Jorgensen, Wavelets through A Looking Glass-The World of the Spectrum (Birkhäuser, Boston, 2002).

[42] K. G. Wilson, Phys. Rev. D 10, 2445 (1974).

[43] K. G. Wilson, Phys. Rep. 23, 331 (1976).

[44] R. Haag, Phys. Rev. 112, 669 (1958).

[45] D. Ruelle, Helv. Phys. Acta 35, 147 (1962).

[46] R. Jost, The General Theory of Quantized Fields (AMS, Providence, 1965).

[47] W. Du, Y. Li, X. Zhao, G. A. Miller, and J. P. Vary, Phys. Rev. C 101, 035202 (2020).

[48] R. Haag, Mat-Fys. Medd. K. Danske Vidensk. Selsk. 29, 1 (1955).

[49] T.-M. Yan, Phys. Rev. D 7, 1780 (1973).

[50] N. Nakanishi and H. Yabuki, Lett. Math. Phys. 1, 371 (1977).

[51] N. Nakanishi and K. Yamawaki, Nucl. Phys. B122, 15 (1977).

[52] F. Lenz, Nucl. Phys. B, Proc. Suppl. 90, 46 (2000).

[53] P. Ullrich and E. Werner, J. Phys. A 39, 6057 (2006).

[54] J. Collins, arXiv:1801.03960v1.

[55] M. Herrmann and W. N. Polyzou, Phys. Rev. D 91, 085043 (2015).

[56] G. Beylkin, SIAM J. Numer Anal. 29, 1716 (1992). 
[57] G. Beylkin and N. Saito, Wavelets, their Autocorrelation Functions, and Multi-Resolution Representation of Signals, edited by D. P. Casasent, Proc. SPIE: Intelligent Robots and Computer Vision XI: Biological, Neural Net, and 3D Methods, Vol. 1826 (SPIE, Boston, 1992), p. 39.
[58] N. Saito and G. Beylkin, IEEE Trans. Signal Process. 41, 3584 (1993).

[59] A. Ben-Israel and T. N. E. Greville, Generalized Inverses, Theory and Applications (Springer, New York, 2003). 